\title{
Genome-wide histone acetylation analysis reveals altered transcriptional regulation in the Parkinson's disease brain
}

\author{
Lilah Toker ${ }^{1,2+}$, Gia T. Tran ${ }^{1,2 \dagger}$, Janani Sundaresan ${ }^{1,2}$, Ole-Bjørn Tysnes ${ }^{1,2}$, Guido Alves ${ }^{3,4}$, Kristoffer Haugarvoll ${ }^{1,2}$,
} Gonzalo S. Nido ${ }^{1,2}$, Christian Dölle ${ }^{1,2}$ and Charalampos Tzoulis ${ }^{1,2^{*}}$ (i)

\begin{abstract}
Background: Parkinson's disease (PD) is a complex, age-related neurodegenerative disorder of largely unknown etiology. PD is strongly associated with mitochondrial respiratory dysfunction, which can lead to epigenetic dysregulation and specifically altered histone acetylation. Nevertheless, and despite the emerging role of epigenetics in age-related brain disorders, the question of whether aberrant histone acetylation is involved in PD remains unresolved.
\end{abstract}

Methods: We studied fresh-frozen brain tissue from two independent cohorts of individuals with idiopathic PD $(n=28)$ and neurologically healthy controls $(n=21)$. We performed comprehensive immunoblotting to identify histone sites with altered acetylation levels in PD, followed by chromatin immunoprecipitation sequencing (ChIPseq). RNA sequencing data from the same individuals was used to assess the impact of altered histone acetylation on gene expression.

Results: Immunoblotting analyses revealed increased acetylation at several histone sites in PD, with the most prominent change observed for H3K27, a marker of active promoters and enhancers. ChIP-seq analysis further indicated that H3K27 hyperacetylation in the PD brain is a genome-wide phenomenon with a strong predilection for genes implicated in the disease, including SNCA, PARK7, PRKN and MAPT. Integration of the ChIP-seq with transcriptomic data from the same individuals revealed that the correlation between promoter H3K27 acetylation and gene expression is attenuated in PD patients, suggesting that H3K27 acetylation may be decoupled from transcription in the PD brain. Strikingly, this decoupling was most pronounced among nuclear-encoded mitochondrial genes, corroborating the notion that impaired crosstalk between the nucleus and mitochondria is involved in the pathogenesis of PD. Our findings independently replicated in the two cohorts.

(Continued on next page)

\footnotetext{
*Correspondence: charalampos.tzoulis@uib.no; charalampos.tzoulis@helsebergen.no

'Lilah Toker and Gia T. Tran contributed equally to this work.

${ }^{1}$ Neuro-SysMed Center of Excellence for Clinical Research in Neurological

Diseases, Department of Neurology, Haukeland University Hospital, 5021 Bergen, Norway

${ }^{2}$ Department of Clinical Medicine, University of Bergen, Pb 7804, 5020 Bergen, Norway

Full list of author information is available at the end of the article
}

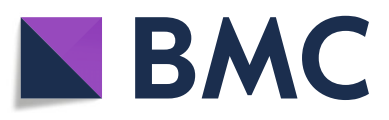

(c) The Author(s). 2021 Open Access This article is licensed under a Creative Commons Attribution 4.0 International License, which permits use, sharing, adaptation, distribution and reproduction in any medium or format, as long as you give appropriate credit to the original author(s) and the source, provide a link to the Creative Commons licence, and indicate if changes were made. The images or other third party material in this article are included in the article's Creative Commons licence, unless indicated otherwise in a credit line to the material. If material is not included in the article's Creative Commons licence and your intended use is not permitted by statutory regulation or exceeds the permitted use, you will need to obtain permission directly from the copyright holder. To view a copy of this licence, visit http://creativecommons.org/licenses/by/4.0/ The Creative Commons Public Domain Dedication waiver (http://creativecommons.org/publicdomain/zero/1.0/) applies to the data made available in this article, unless otherwise stated in a credit line to the data. 
(Continued from previous page)

Conclusions: Our findings strongly suggest that aberrant histone acetylation and altered transcriptional regulation are involved in the pathophysiology of PD. We demonstrate that PD-associated genes are particularly prone to epigenetic dysregulation and identify novel epigenetic signatures associated with the disease.

Keywords: H3K27, Epigenetics, Neurodegeneration, ChIP-seq, Sirtuin, Mitochondria, Gene expression

\section{Background}

Parkinson's disease (PD) is the second most common neurodegenerative disorder, affecting $\sim 1.8 \%$ of the population above the age of 65 years [1]. The neuropathological hallmark of PD is the progressive loss of dopaminergic neurons in the substantia nigra pars compacta $(\mathrm{SNc})$ in the presence of $\alpha$-synuclein-positive inclusions termed Lewy pathology. Additional neurodegenerative changes occur in multiple regions of the nervous system, including several brainstem nuclei, the olfactory bulb, hippocampus, amygdala and the neocortex, as well as the autonomic and enteric nervous systems [2].

The etiology of PD remains largely unknown. While monogenic forms of PD exist, they are rare, generally accounting for less than $5-10 \%$ of all cases in most populations. The vast majority of patients have "idiopathic PD", which is of complex etiology [3]. Genome-wide association studies (GWAS) have identified multiple risk loci associated with idiopathic PD. These make a relatively small collective contribution to the disease risk, however, and have not been linked to specific molecular mechanisms that could be targeted therapeutically [4].

Epigenetic modifications, such as DNA methylation and histone acetylation, modulate gene expression without altering the underlying DNA sequence. Dysregulation of histone acetylation has been previously linked to aging [5] and neurodegeneration [6] and changes in histone acetylation at specific genomic regions have been reported in Alzheimer's disease [7, 8]. Despite the emerging role of epigenetics in age-related brain disorders, only a few small-scale studies of histone acetylation in PD have been reported to date, providing inconclusive results [9-11]. Moreover, the genomic landscape of histone acetylation in PD has never been assessed.

Here, we report the first genome-wide study of histone acetylation in the PD brain, based on a population-based cohort from the Norwegian Park West study (PW) [12]. Our findings replicate in an independent cohort of subjects from the Netherlands Brain Bank (NBB) and reveal genome-wide dysregulation of H3K27 acetylation in the PD brain.

\section{Methods}

\section{Cohorts and tissue}

Brains were collected at autopsy and split sagittally along the corpus callosum. One hemisphere was fixed whole in formaldehyde for $\sim 2$ weeks, and the other coronally sectioned and snap-frozen in liquid nitrogen. Samples from the frozen hemisphere were included in this study. Prefrontal cortex (Brodmann area 9) was obtained from two independent cohorts. The discovery cohort (ParkWest, or PW cohort) comprised individuals with idiopathic PD $(n=17)$ from the ParkWest study, a prospective population-based cohort which has been described in detail [12], and neurologically healthy controls $(n=15)$ from the Neuromics Biobank for Aging and Neurodegeneration. For a subset of the PW cohort (PD: $n=7$, Control: $n=7$ ), tissue samples from the striatum (putamen) and cerebellar cortex were also included. All individuals of the PW cohort had undergone wholeexome sequencing [13] and pathogenic mutations in genes implicated in Mendelian PD and other monogenic neurological disorders had been ruled-out. The replication cohort comprised individuals with idiopathic PD $(n=10)$ and neurologically healthy controls $(n=11)$ of Dutch origin, from the Netherlands Brain Bank (https:// www.brainbank.nl/). Demographic information and experimental allocation of all samples is shown in Supplementary Table S1. For each experiment, there were no significant differences for sex, age, or postmortem interval (PMI) between the compared groups. A complete list of the medication used by the individuals with PD during at least the last 12 months before death can be found in Supplementary Table S1. Individuals with PD fulfilled the National Institute of Neurological Disorders and Stroke [14] and the U.K. PD Society Brain Bank [15] diagnostic criteria for PD at their final visit. All cases showed neuropathological changes consistent with PD (Supplementary Table S1).

\section{Immunoblotting}

Three independently dissected samples of brain tissue (replicates) were analyzed from each individual. Immunoblots were repeated at least 2 times for each marker. To normalize the histone acetylation signal to histone quantity, each acetylation blot was stripped twice. Complete stripping was confirmed by incubation of the membranes with secondary antibodies only and ECL. To exclude potential bias due to incomplete stripping, we performed additional experiments where acetylated and total histone markers for each histone modification were assessed in parallel on different blots. To estimate the 
acetylated fraction of each histone marker, we calculated the signal ratio of acetylated marker to total histone protein. This ratio is referred to as the "acetylation fraction" for each histone marker. Detailed description is provided in the Supplementary material, methods section. Comparison of immunoblot densitometric values between groups was performed using linear regression for panacetylation and linear mixed-models for all other markers. Linear mixed-models analysis was chosen in order to account for intraindividual random variability. Subjects were specified as random effects and disease state, sex, age of death, PMI and oligodendrocyte MSP were used as fixed effects. The error distribution was assumed normal, and solution to the mixed models was obtained using the maximum likelihood estimation method. The mixed-models analyses were implemented in SPSS version 22.0.0.

\section{Chromatin Immunoprecipitation sequencing (ChIP-seq)}

Approximately $200 \mathrm{mg}$ of frozen PFC tissue per sample was used for ChIP-seq. Briefly, the tissue was crosslinked using formaldehyde, lysed using a Dounce homogenizer and sonicated to obtained chromatin fragment of 300500 bp. Chromatin was immunoprecipitated using an antibody against acetylated H3K27. Protein-DNA complexes were recovered using protein A agarose beads, de-crosslinked and DNA was purified by phenolchloroform extraction and ethanol precipitation. Following quantitative PCR, libraries were built and sequenced on Illumina's NextSeq 500 (75 base pair reads, single end). Detailed description is provided in the Supplementary material, methods section.

\section{ChIP-seq quality control and filtering}

The analysis of the ChIP-seq data was performed in accordance with ENCODE ChIP-seq standards https://www.encodeproject.org/chip-seq/histone/ \#standards. Raw FASTQ files were trimmed using Trimmomatic version 0.38 [16] with options ILLUMINACLIP:TruSeq2-SE.fa:2:30:10 LEADING:3 TRAILI NG:3

SLIDINGWINDOW:4:15 MINLEN:25. Assessment of FASTQ files was carried out with FastQC version 0.11.8 [https://www.bioinformatics.babraham.ac.uk/projects/ fastqc] before and after trimming. Reads were aligned to the GRCh37 human genome using bwa version 0.7.17 [17]. Reads with mapping quality $<30$ were discarded using samtools version 1.9 [https://www.htslib.org/] and duplicated reads removed using MarkDuplicates from Picard Tools version 2.18.27 [https://broadinstitute. github.io/picard/]. BAM files were converted into BED files and sorted.

\section{Peak calling and read counting}

Peak calling procedure was carried out in two complementary approaches: 1) Per group (PD or controls): the peaks are called separately for each group, aggregating the relevant samples and inputs. While this approach is not optimal for differential peak calling analysis as it requires harmonization of peaks between the two groups, it can be used to compare the total genome coverage as well as unique $\mathrm{H} 3 \mathrm{~K} 27 \mathrm{ac}$ genomic regions in each group. 2) All samples combined: the peaks are called by aggregating all samples and input controls. This approach does not allow identification of unique genomic regions however, it provides a better identification of consensus peaks, and thus, was used for differential peak analysis. The resulting peak-sets were filtered using the ENCODE black listed regions and only peaks within canonical chromosomes were kept for downstream analyses. Sample-specific enrichment on the identified peaks was performed using featureCounts version 1.6.4 [18] with default parameters. For both the individual group and all samples combined approaches, peaks with non-adjusted $p$-value $>10^{-7}$ were excluded from the analysis. Reads inside peaks (RiP) were quantified using "featureCounts" program from the "subread" package v2.0.0. Additional details are provided in the Supplementary material, methods section.

\section{Calculation of marker site profiles (MSP)}

In order to identify brain cell-type specific H3K27ac regions we first analyzed H3K27ac ChIP-seq data from $\mathrm{NeuN}^{+}$and $\mathrm{NeuN}^{-}$brain cells [19]. Cell-type based broadPeaks (CellType_peak-set), count matrixes and metadata files were downloaded from https://www. synapse.org/\#!Synapse:syn4566010. Differential regions between $\mathrm{NeuN}^{+}$and $\mathrm{NeuN}^{-}$cells were calculated using "DESeq2" R package [20], including chromatin amount, library batch, sex, hemisphere, age and $\mathrm{pH}$ as covariates in the model. Peaks were defined as cell-type specific differentially acetylated regions (DARs) if they met the following criteria: a) |fold change| $>4$ and b) mean count $>$ 1000. Peaks were annotated to all genes for which they intersected a region between $5 \mathrm{~kb}$ upstream from their transcription start site (TSS) to the end of their 5'UTR using "annotatr" R package [21], based on UCSC hg19 genome assembly. Genes with DARs were then itersected with expression-based cortical marker gene lists based on NeuroExpresso database [22]. The DARs were next reassigned to specific cell-types if they were annotated to genes defined as cell-type specific based on NeuroExpresso. For example, all DARs annotated to $M B P$, defined as oligodendrocyte marker gene based on NeuroExpresso, were defined as oligodendrocyte marker sites (MSS). In the next step, we quantified the reads from our samples in the CellType_peak-set. The 
corresponding RiP were then converted to counts per million (CPM) and transformed to $\log 2(\mathrm{CPM}+1)$. Next, for each cell-type specific MSS, we performed principal component analysis based on the relevant peaks as described in [22]. Marker Site Profiles (MSP) were defined as the scores of the samples in the first principal component, transformed to $[0,1]$ range for visualization purposes. Detailed description of the analysis is provided in the Supplementary material, methods section.

\section{Identification of differentially acetylated regions}

Differential peak analysis was performed using "nbinomWaldTest" function from "DESeq2" $\mathrm{R}$ package. Dispersions were calculated by using "estimateDispertions" function from "DESeq2" R package, by setting the fitType parameter to "local". Normalization factors calculated by the "estimateSizeFactors" function were replaced with the housekeeping normalization factor based on our manually selected housekeeping gene set $(A C T B, G A P D H, U B C)$. Peak significance was determined using DESeq2 "results" function, setting the cutoff for adjusted $p$-value to 0.05 , and independentFiltering = $\mathrm{T}$. Detailed description of the analysis is provided in the Supplementary material, methods section.

\section{Association of H3K27 hyperacetylation with p300 and non-SIRT1 HDAC binding}

ChIP-seq data (wgEncodeRegTfbsClusteredV3.bed) was obtained from the Encyclopedia of DNA Elements (ENCODE) project [23]. The data was available for p300 and the following HDACs: HDAC1, HDAC2, HDAC6, HDAC8 and SIRT6 We first quantified the number of binding sites for each protein in our peaks using the "findOverlaps" function from the "GenomicRanges" R package [24]. For each peak, the HDAC binding sites were then summed up to represent the non-SIRT1 HDAC_binding. Peaks that had no binding sites for either of the proteins were excluded from downstream analyses. For the gene level analysis, the number of binding sites in each peak was collapsed to a gene level.

Difference between DARs and non DARs was assessed by comparing the value of $\log \frac{p 300_{\text {Binding }}+0.01}{H D A C_{\text {binding }}+0.01}$ of the peaks using Kolmogorov-Smirnov test (all genes) or Wilcoxson's rank sum test (PD_implicated genes) using "ks.test" and "wilcox.test" functions from R "stats" package. For linear model, we first obtained one-sided hyperacetyla-

tion $p$-value $\left(\mathrm{p}_{\text {hyper }}\right)$ for each peak as followed: $P_{\text {hyper }}$ $=\left\{\begin{array}{c}1-p / 2, L F C<0 \\ p / 2, L F C \geq 0\end{array}\right.$. The association of hyperacetylation with p300_binding and HDAC binding was then assessed by the linear model: $-\log 10\left(p_{\text {hyper }}\right) \sim p 300_{\text {bind- }}$ ing $+H D A C_{\text {binding }}+$ PDgene where PDgene indicates whether a peak is annotated to PD_implicated gene or not.

\section{Association of PD_implicated genes and EGL with altered H3K27 acetylation}

Effective gene length (EGL) was defined as sum of length of all regions annotated to the gene (including $1 \mathrm{~Kb}$ upstream to TSS). We assessed the association between altered H3K27 acetylation defined as - $\log 10$ (metaP) with being annotated to a PD_implicated gene (PDgene), EGL and the total number of common regions aligned to the gene using a linear model. The total number of common regions in a gene was included as a covariate in the model. Namely, for a common region i, annotated to gene $\mathrm{j}$, the $-\log ($ metaP) value was modeled as: $-\log$ $10\left(\right.$ metaP $\left._{i j}\right) \sim$ PDgene $_{j}+\log 10\left(E G L_{j}\right)+\sum$ CommonRe- $^{\prime}$

gions $_{j}$. For the analysis was performed in each cohort separately, metaP was replaced with cohort-specific $p$ value, and the total number of common regions was replaced with the total number of regions (peaks) annotated to the gene in the cohort-specific peak-set.

\section{Transcription factor enrichment analysis}

The genomic sequence of hypo- and hyper-acetylated regions were repeat masked, and the enrichment of transcription factor binding motifs (TFBM) was assessed using the Analysis Motif of Enrichment (AME tool) [25] from the MEME Suite [26] v5.3.2, by setting the fraction of maximum log-odds to 0.75, and using human (HOCOMOCO v11) motif database [27]. The enrichment was assessed using Fisher's exact test, with length-matched nondifferentially acetylated regions set as the background.

\section{RNA-seq analysis}

Total RNA was extracted from prefrontal cortex tissue homogenate for all samples for which ChIP-seq was performed and sequenced following ribosomal RNA depletion A detailed description of the RNA-seq analysis is provided in the Supplementary material, methods section and is reported elsewhere [28]. Relative cell abundance was estimated using cell-type specific marker genes, as previously described [22, 29]. Since significant group effect was found for oligodendrocytes and microglia estimates in PW (Wilcoxon's $p=2.6 \times 10^{-4}$ ) and NBB (Wilcoxon's $p=0.009$ ) cohorts respectively, these cell types were included at covariates in the analysis of both cohorts. Count matrix and the code necessary to reproduce these results are available on https://git.app. uib.no/neuromics/cell-composition-rna-pd.

\section{Promoter H3K27ac - expression correlation}

ChIP-seq and RNA-seq counts were first adjusted to the relevant covariates (age, sex, PMI, batch, oligodendrocyte estimates and microglia estimates). The adjustment 
was done by re-fitting the counts using the coefficients calculated by DESeq2 $\mathrm{R}$ function with adjusted model matrix in which categorical covariates were set to 0 , and the continuous covariates to the mean of the original values. Next, for each gene, Pearson's correlation between the adjusted ChIP-seq/RNA-seq seq counts was computed using "cor.test" $\mathrm{R}$ function, in each group (controls or PD subjects) separately. Only peaks annotated to promoter regions of the genes were included in the analysis. When multiple promoter peaks were defined for a gene, the correlation was calculated for each promoter separately, and the highest correlation was kept. This step was performed independently for each group. Since not all genes are expected to exhibit a good correspondence between promoter $\mathrm{H} 3 \mathrm{~K} 27 \mathrm{ac}$ state and expression level due to existence of multiple levels of regulation, we compared the distribution of correlations in each group for different thresholds of absolute correlation in either of the groups (e.g., cor $>|0.1|$ means that only genes with absolute correlation $>0.1$ in at least one of the groups are included in the analysis). Group comparison was performed using Wilcoxon rank sum test, using "wilcox.test" function from R "stats" function. Functional enrichment analysis was performed using the gene score resampling method implemented in the ermineR package version 1.0.1 [30], an $R$ wrapper package for ermineJ [31], setting the value of "BigIsBetter" to FALSE, with the complete Gene Ontology (GO) database annotation [32]. For the enrichment of genes based on the decoupling levels, gene scores were defined as $\Delta C o r=\operatorname{Cor}_{P D}-$ Cor $_{\text {Control }}$. For enrichment of genes based on the lowest correlation in PD group, gene scored were defined as the correlation values in the PD group.

\section{Data and code availability}

The $\mathrm{R}$ code necessary to reproduce the results presented in the paper is available on https://github.com/ltoker/ ChIPseqPD. Raw counts for the ChIP-seq analyses are available in the same repository (https://github.com/ ltoker/ChIPseqPD/tree/master/data). UCSC genome browser tracks showing the H3K27ac peak-sets based on PW and NBB cohorts can be accessed at https:// genome-euro.ucsc.edu/s/ltoker/H3K27ChIP_PD. Additional R code to reproduce the RNA-seq analyses is available on https://git.app.uib.no/neuromics/cellcomposition-rna-pd. The corresponding raw data counts are included in the repository (https://git.app.uib.no/ neuromics/cell-composition-rna-pd/tree/master/Data).

\section{Results}

Increased histone acetylation and histone protein levels in the PD brain

We first assessed global protein acetylation in the prefrontal cortex (PFC) of 13 individuals with PD and 13 demographically matched controls from the PW cohort by immunoblot analysis using an antibody against acetylated lysine (Fig. 1a, Supplementary Table S1). We concentrated on PFC because, while this area is typically affected in PD [33-35], it is generally less confounded by altered cell composition due to neurodegeneration and neuroinflammation [2]. Linear regression analysis of the densitometric measurements, adjusted for age, sex and PMI, indicated an increased acetylation signal at $17 \mathrm{kDa}$, consistent with the molecular weight of core histone proteins ( $p=0.014$, Fig. 1a).

To identify histone sites contributing to the hyperacetylation observed in our samples and select a specific site for downstream analyses, we assessed the acetylation state at multiple sites on the four core histones in $7 \mathrm{PD}$ and 7 control samples showing a prominent difference in the pan-acetylation analysis (Fig. 1b, Supplementary Table S1). The acetylated fraction of each histone site and the total histone quantity were analyzed using linear mixed models with gender, age and PMI as fixed effects and subject and blot number as random effects. These analyses indicated a significant increase in H2BK15ac $\left(p=1.4 \times 10^{-5}\right), \quad$ H3K9/14 ac $\left(p=3.9 \times 10^{-4}\right), \quad$ H3K27ac $\left(p=4.5 \times 10^{-8}\right)$, H3K56ac $(p=0.01)$ and H4K12ac $(p=$ $\left.5.0 \times 10^{-3}\right)$ in PD. No significant difference was found for H2AK5, H4K5, H4K16 or $\alpha$-tubulin, used as a nonhistone control (Fig. 1b-c, Supplementary Table S2). In addition, we found increase in the total quantity of the four histone proteins (H2A: $p=2.7 \times 10^{-5}$, H2B: $p=$ $\left.1.1 \times 10^{-8}, \mathrm{H} 3: p=2.0 \times 10^{-7} ; \mathrm{H} 4: p=3.9 \times 10^{-18}\right)$.

To control that our findings were not driven by differences in cell type composition, we carried out immunoblot analyses of protein markers for neurons (NeuN), astrocytes (GFAP), oligodendrocytes (CNP1), microglia (CX3CR1) and total number of nuclei (NUP188), in the same samples used for acetylation assessment. Statistical analyses of these data indicated increase in CNP1 $(p=$ $3.9 \times 10^{-5}$, Fig. 1d, Supplementary Table S2), but no changes in the other markers. We thus repeated the histone acetylation analyses adding CNP1 as a covariate to the statistical model. As shown in Supplementary Table $\mathrm{S} 2$, all of the indicated hyperacetylated sites remained significant after CNP1 adjustment.

Similar to findings from PFC, H3K27 hyperacetylation was also observed in the PD striatum $(p=0.034)$ and cerebellum $(p=0.016)$, areas typically spared by neuronal loss [2], but exhibiting disease-related pathology, including Lewy pathology $[36,37]$ and mitochondrial respiratory deficiency [38] (Fig. 2, Supplementary Table S2).

\section{Histone hyperacetylation in the PD brain is accompanied by upregulation of sirtuin protein levels}

Histone hyperacetylation can be induced by mitochondrial respiratory complex-I deficiency $[10,39,40]$, a 


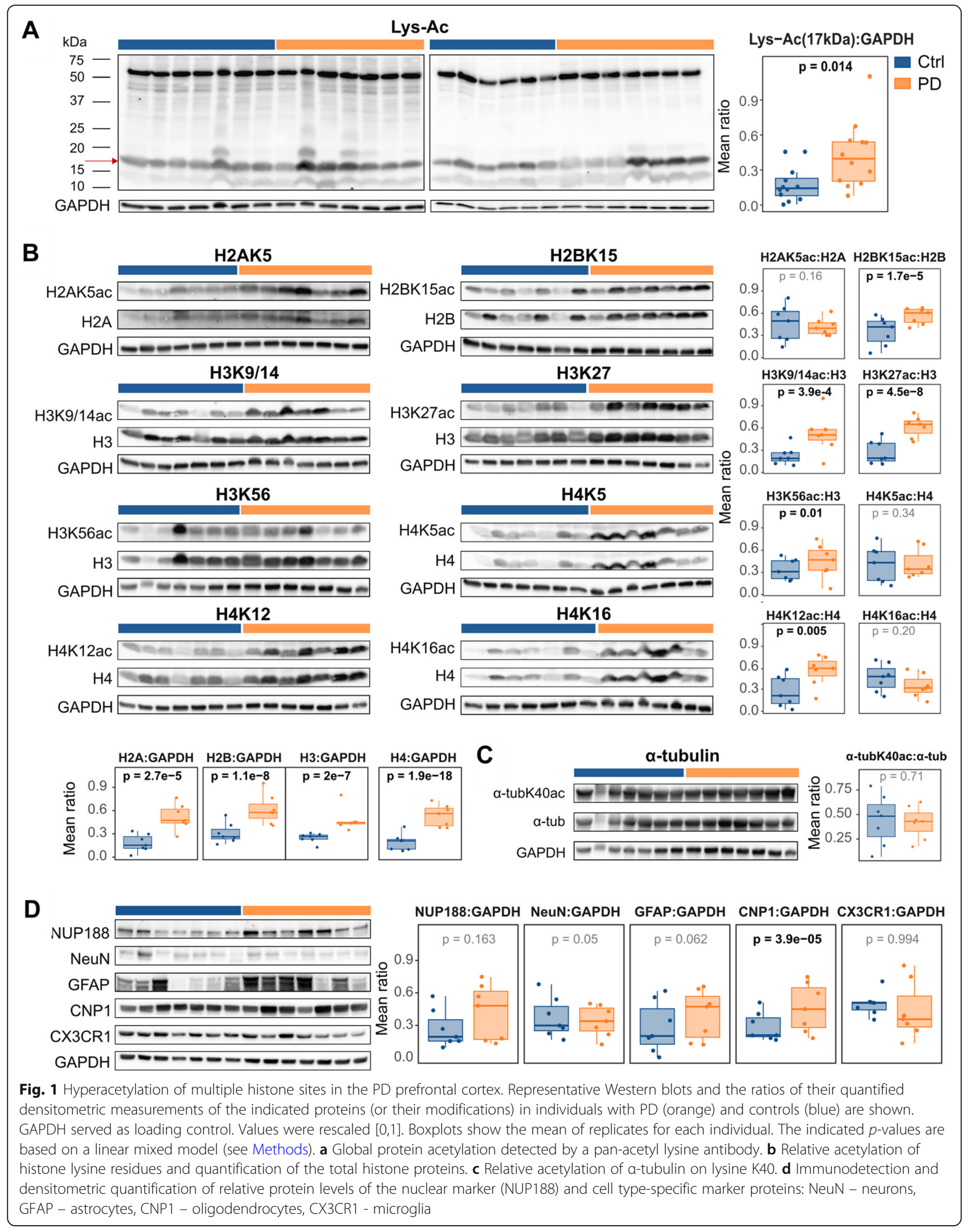



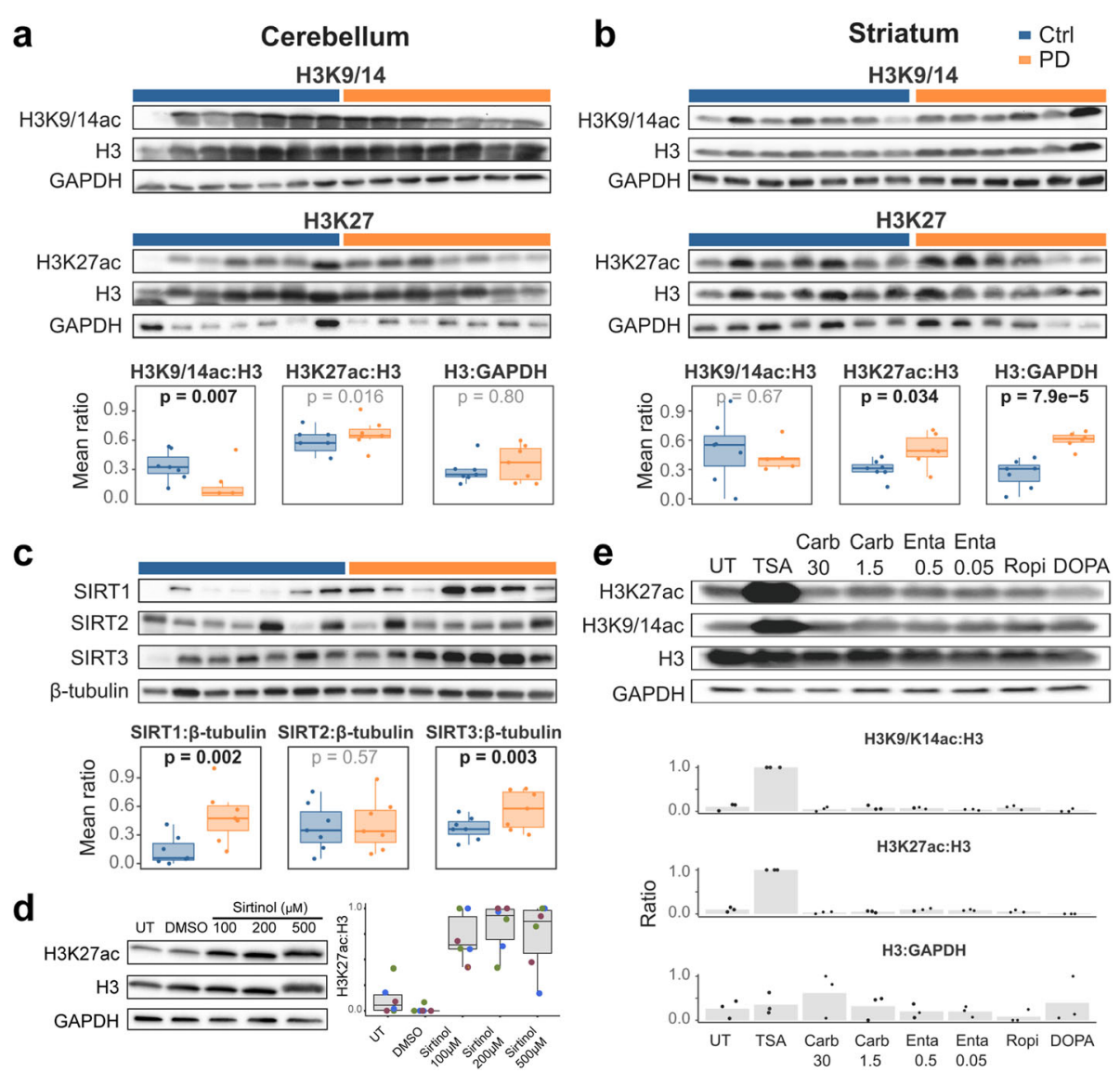

Fig. 2 Altered $\mathrm{H} 3$ acetylation occurs in multiple brain regions, is accompanied by sirtuin upregulation and is not induced by anti-Parkinson drugs in-vitro. a-c Representative Western blots and quantification plots showing the ratios of quantified densitometric measurements of the indicated proteins in individuals with PD (orange) and controls (blue). GAPDH and $\beta$-tubulin serve as loading controls. Values were rescaled [0,1]. Boxplots show the mean of replicates for each individual. The indicated $p$-values are based on a linear mixed model (see Methods). $\mathbf{a}, \mathbf{b}$ Acetylated H3K9/ 14 and H3K27 and total histone protein H3 levels in the cerebellum (a) and striatum (b) from individuals with PD and controls. c Sirtuin proteins in the prefrontal cortex of PD and controls. d Acetylated H3K27 in SH-SY5Y cells, untreated (UT) or treated with different concentrations of the sirtuin inhibitor Sirtinol, or the solvent (DMSO) for $6 \mathrm{~h}$. A representative blot is shown. Boxplots show the quantified ratios of three biological replicates (indicated in different colors) carried out in duplicates, rescaled [0,1]. e Acetylated H3K9/14, H3K27 and total H3 protein in differentiated SH-SY5Y-derived dopaminergic neuron-like cells treated with the histone deacetylase inhibitor Trichostatin A (TSA) or anti-Parkinson drugs used by the PW patients during their last year of life. The numbers indicate the concentration (in $\mu \mathrm{M}$ ) of the drug. Carb: Carbidopa; Enta: Entacapone; Ropi: Ropinirole; DOPA: L-Dopa. UT: untreated control. A representative blot is shown. Bar plots show the mean quantified ratios of three biological replicates (rescaled $[0,1])$. Dots indicate the values of each of the individual replicates

pathological feature found throughout the PD brain, including the PFC, striatum and cerebellum [38]. This effect is thought to be mediated by decreased $\mathrm{NAD}^{+} /$ $\mathrm{NADH}$ ratio, attenuating the activity of $\mathrm{NAD}^{+}$dependent class III histone deacetylases known as sirtuins [39, 41-44]. Direct confirmation of this mechanism in our samples would require a reliable determination of their intracellular $\mathrm{NAD}^{+}$, which was not feasible due to rapid postmortem degradation [45]. However, it was previously reported that decreased sirtuin activity caused by $\mathrm{NAD}^{+}$deficiency is accompanied by increased protein quantity $[46,47]$. Thus, we assessed the protein levels of SIRT1, SIRT2 and SIRT3, localized to the nucleus, cytosol and mitochondria, respectively, as surrogates for their activity. We were specifically interested in these proteins, because they have been linked to aging and neurodegeneration [41]. Immunoblot analyses of the 14 samples assessed for histone acetylation, indicated a significant upregulation of SIRT1 $\left(p=2.0 \times 10^{-3}\right)$ and SIRT3 $\left(p=3.0 \times 10^{-3}\right)$ in PD. No difference was detected for SIRT2 (Fig. 2c, Supplementary Table S2), in line with the unaltered acetylation state of its target, $\alpha$-tubulin (Fig. 1c). Moreover, treatment of SHSY5Y cells with the sirtuin inhibitor sirtinol led to a substantial hyperacetylation of H3K27, in comparison to control cells (Fig. 2d), indicating that sirtuins can indeed modulate the acetylation of this residue. 


\section{Histone hyperacetylation is not induced by anti-Parkinson drugs in cellulo}

We next investigated whether the observed altered histone acetylation in PD could result from treatment with anti-Parkinson agents. To test this, we exposed fully differentiated SH-SY5Y cells to the drugs used by most individuals with PD from the PW cohort during their last year of life (L-DOPA, carbidopa, entacapone and ropinirole), and monitored acetylation of $\mathrm{H} 3 \mathrm{~K} 9 / 14$ and H3K27 by immunoblot (Supplementary methods). These analyses indicated no effect for any of the tested antiParkinson drugs on histone acetylation in our model (Fig. 2e). Nevertheless, these findings cannot completely rule out the possibility that altered histone acetylation can be, at least partially, induced by drug-related effects in the human brain over a prolonged period of time.

\section{Acetylated H3K27 exhibits higher genomic occupancy in PD}

We next carried out ChIP-seq analysis of acetylated H3K27 (H3K27ac) in all PFC samples from the PW cohort $(n=28)$, Supplementary Table S1). First, we characterized the genomic distribution of H3K27ac in the PD and control groups. For this purpose, we identified H3K27ac enriched genomic regions (peaks) in each group separately (see Methods). In line with the increased H3K27ac signal observed in the immunoblot analysis, a higher number of peaks $(146,763$ vs. 135,662), as well as a higher proportion of group-unique peaks $(32.2 \%$ vs. $13.2 \%)$, and wider genome coverage (10.6\% vs. 9.11\%) were found in the PD- compared to the control group (Fig. 3a, Supplementary Table S3). We then compared the distribution of genomic annotations and detection $p$-values of overlapping and unique peaks between the PD and control groups. In both groups, unique peaks occurred more frequently in intergenic and intronic regions than in promoter and exonic regions (Supplementary Fig. S1a, b). Since unique peaks were also characterized by significantly higher detection $p$-values, compared to the overlapping peaks (Fig. S1c, d), we believe that most of these represent "noisy" H3K27 acetylation.

\section{ChIP-seq data corroborates H3K27 hyperacetylation in the PD brain}

Next, we redefined H3K27ac peaks by identifying H3K27ac enriched regions by aggregating all PW samples (see Methods). The final peak-set consisted of 160, 521 peaks, covering $11.6 \%$ of the genome and distributed over all 22 autosomes and both sex chromosomes (median peak size, $817 \mathrm{bp}$, Fig. 3d). All downstream analyses were performed based on this peak-set, henceforth referred to as PW_peak-set.
ChIP-seq data reflects the abundance and genomic distribution of the target protein (or protein modification) which can vary substantially between samples [48]. Based on the observed increase in H3K27ac fraction in the immunoblot analysis, we anticipated an increase in overall H3K27ac ChIP-seq signal in PD. Thus, we reasoned that the most suitable approach for normalization of our data would be using reads in peaks (RiP) annotated to house-keeping genes, as previously proposed [48]. We modified this approach by manually selecting peaks annotated to known housekeeping genes that exhibit a saturated peak signal covering most of the gene body (Supplementary Fig. S2). The rationale here was that these genes should be saturated with histone acetylation and are, therefore, expected to exhibit little biological variation.

To validate our normalization approach, we performed additional H3K27ac immunoblot and ChIP-seq analyses in samples from 55 individuals (including the study subjects, Supplementary Table S1) and tested the correlation between the immunoblot and ChIP-seq data normalized by different approaches. As shown in Supplementary Fig. S3, the highest correlation between immunoblotting and ChIP-seq data was observed when the total number of RiP was normalized to our manually chosen peaks. Importantly, comparison of normalized RiP between PD and control samples corroborated the finding of general H3K27 hyperacetylation in PD (Supplementary Fig. S3).

\section{Cell composition is a major source of variation in bulk tissue H3K27ac ChIP-seq data}

Since different cell-types exhibit distinct epigenetic landscapes [19], bulk tissue data must be evaluated in the context of the underlying cellular composition [22, 49, 50]. To estimate the cellular composition in our ChIPseq data, we used an approach similar to the one described by Mancarci et al. [22]. Briefly, we intersected genomic H3K27ac binding sites differentiating between NeuN-positive and NeuN-negative brain cells [19] with the list of brain cell-type specific marker-genes [22], in order to obtain genomic H3K27ac cell-type marker sites. The first principal component of reads aligned to the marker sites was used to obtain Marker Site Profiles (MSPs) and was used as a proxy for the relative abundance of the corresponding cell-types across the samples. We validated this approach in publicly available ChIP-seq data from cortical and cerebellar bulk tissue samples [51], and ChIP-seq data from entorhinal cortex of individuals with Alzheimer's disease and healthy controls [8]. MSP analysis of these data successfully recapitulated the well-known increased abundance of glial cells in cortical vs cerebellar samples [52, 53], and decreased number of neurons in the entorhinal cortex of subjects 


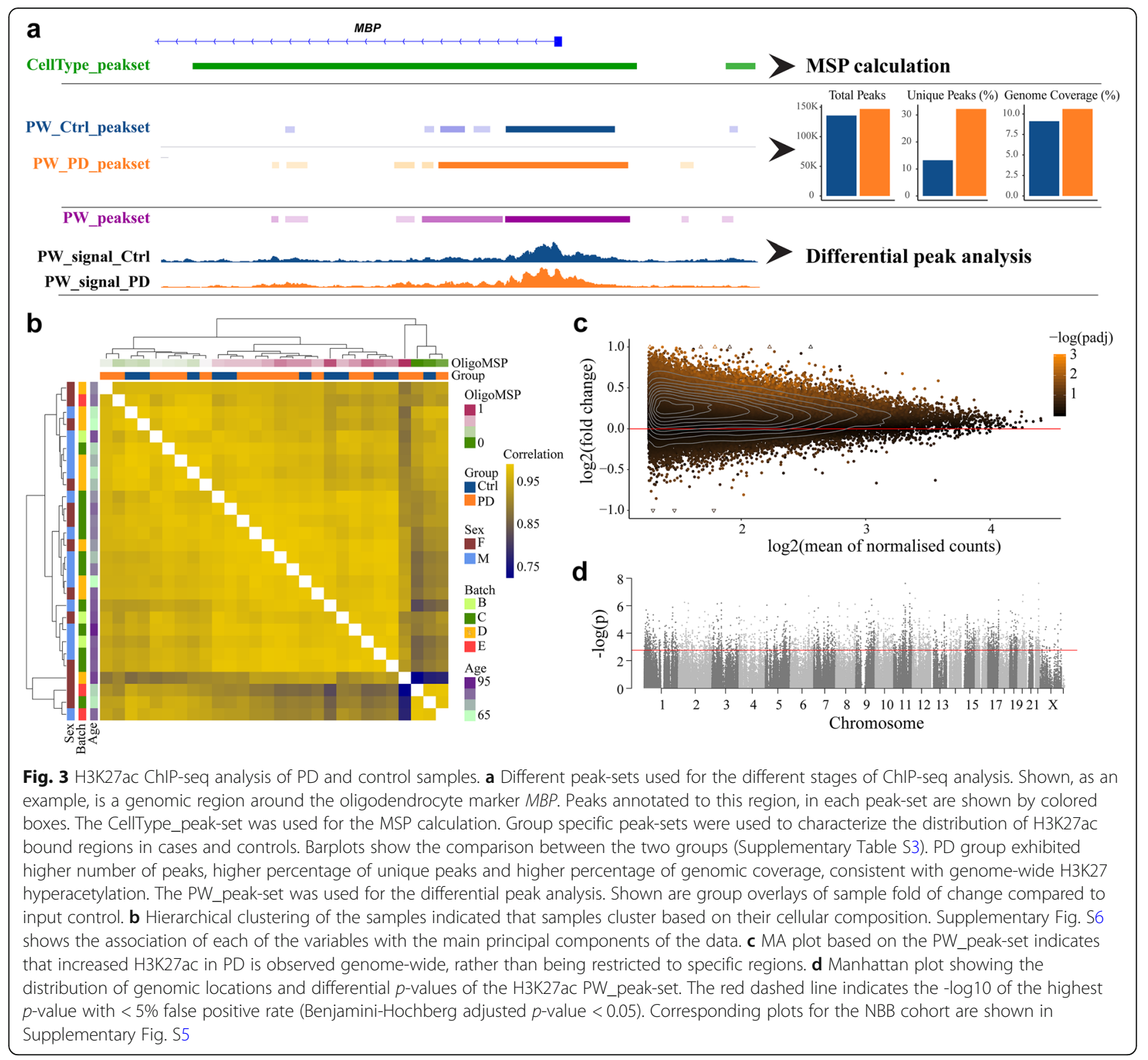

with Alzheimer's disease compared to controls [54, 55] (Supplementary Fig. S4a, b).

Next, we performed MSP analysis in the PW_peak-set data. This analysis indicated no significant differences in cell-type abundance between the PD and control groups (Supplementary Fig. S5). Nevertheless, since the cellular composition of cortical samples can be affected by variable white matter contamination introduced during dissection [28], we assessed the effect of oligodendrocyte MSPs and other experimental and demographic covariates on the ChIP-seq data. Sample-to-sample correlation indicated that the samples clustered mainly based on oligodendrocyte MSPs (Fig. 3b). Accordingly, the first principal component of the data was mostly associated with oligodendrocyte MSP (Supplementary Fig. S6), confirming that oligodendrocyte composition is a major source of variance in our data. Therefore, oligodendrocyte MSPs were accounted for in all downstream analyses.

\section{Hyperacetylation of H3K27 in the PD brain is a genome- wide phenomenon}

We next sought to identify differentially acetylated regions (DARs) for H3K27 between PD and control samples. After filtering (see Methods), 133,716 peaks annotated to 17,182 genes remained for downstream analyses. Five samples (one control and four PD samples) were detected as outliers and excluded from differential peak analysis (Supplementary Table S1). Differential peak analysis was performed using the 
"DESeq2" R package, adjusting for age, sex, batch, PMI, oligodendrocyte MSP and normalization peak ratio. DARs were defined as differentially acetylated peaks with adjusted $p$-value $<0.05$.

Our analysis identified 2877 hyperacetylated and only 14 hypoacetylated PD-associated DARs, annotated to 1434 and 9 genes, respectively. The top-ranked hyperacetylated and hypoacetylated DARs resided within the DLG2 (adjusted $p=9.8 \times 10^{-4}$ ) and PTPRH (adjusted $p$ value $9.0 \times 10^{-3}$ ) genes, respectively, both of which have been previously associated with PD [4,56-58]. The full list of DARs with their annotated genes is provided in Supplementary Table S4. An MA-plot (the log ratio vs. mean count) of all regions included in the analysis indicated a genome-wide trend for H3K27 hyperacetylation in PD (Fig. 3c).

\section{H3K27 hyperacetylation in the PD cortex is replicated in an independent cohort}

To validate our findings, we carried out ChIP-seq in an independent cohort from the Netherlands Brain Bank (NBB, Supplementary Table S1). In agreement with our finding from the PW cohort, we observed an increase in the percentage of unique peaks as well as increased total genomic coverage in the PD group (Supplementary Table S3). The distribution of genomic annotations among the unique and overlapping peaks and the distribution of peak $p$-values were similar between the two cohorts (Supplementary Fig. S1). As in the PW cohort, cellular composition accounted for most of the interindividual variance in this cohort (Supplementary Fig. S6b, S7a).

Differential acetylation analysis corroborated the finding of PD-associated genome-wide H3K27 hyperacetylation observed in the PW cohort (Supplementary Fig. S5b). In total, the analysis identified 2486 hyperacetylated DARs, mapped to 946 genes, and 227 hypoacetylated DARs, mapped to 253 genes (Supplementary Table S5). Strikingly, 275 out of the 946 genes with hyperacetylated DARs and 2 out of 253 genes with hypoacetylated DARs replicated across both cohorts (Fig. 4c, hypergeometric test: $p=2.49 \times 10^{-85}$ and $p=8.2 \times 10^{-3}$, respectively). These 277 genes are henceforth referred to as "replicated genes". The top replicated genes (ranked

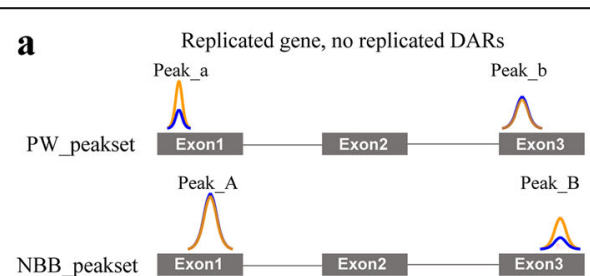

b
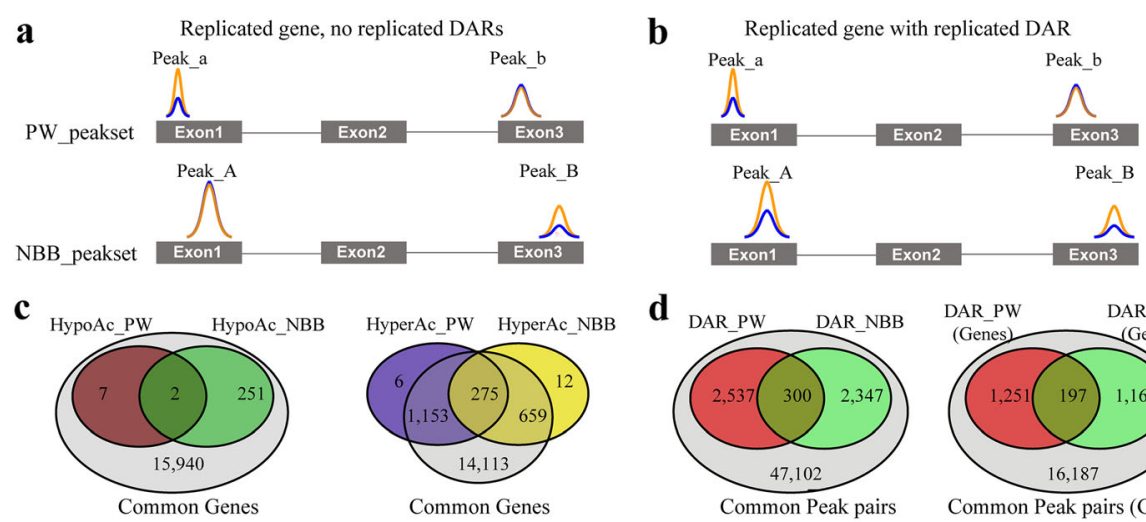

d

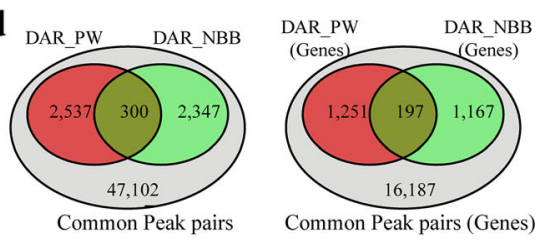

$\mathbf{e}$

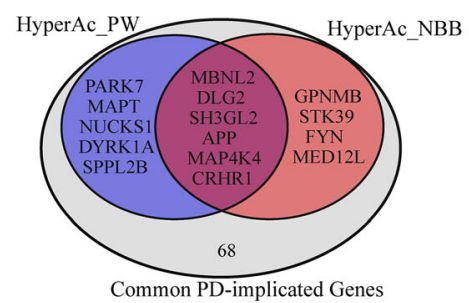

f

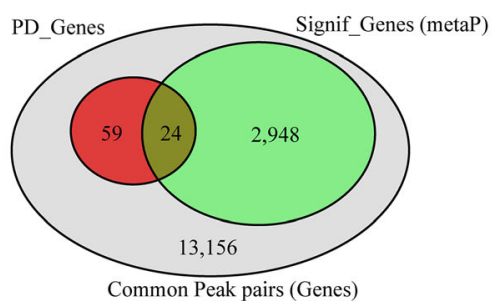

Fig. 4 Replicated genes and replicated DARs. a, $\mathbf{b}$ Schematic representation of two levels of replication between the PW and NBB cohorts H3K27ac peaks are illustrated by colored lines, representing control (blue) and PD (orange) samples, with the height of the peak representing the peak intensity. Shown is a hypothetical gene $X$, with exons represented as grey boxes and introns represented by lines. In both cases, the gene has two pairs of common peaks: Peak_a/A, mapped to the first exon of the gene and Peak_b/B, mapped to the third exon of the gene. a Altered H3K27 acetylation occurs at different regions of gene $X$ in each cohort. Thus, while gene $X$ harbors altered H3K27 acetylation in both cohorts (i.e. gene $X$ is a replicated gene), it does not harbor replicated DARs. $\mathbf{b}$ Altered H3K27 acetylation occurs at the same region of gene $\mathrm{X}$ in both cohorts. In this case, gene $X$ is a replicated gene with a replicated DAR. c Venn diagram showing the number of replicated genes (with or without replicated DARs) between the two cohorts, out of all genes represented in our ChIP-seq data from both cohorts. $\mathbf{d}$ Venn diagrams showing the number of replicated DARs between the two cohorts, out of all common peak pairs (left) and their corresponding annotated genes (right). e Venn diagram showing the overlap of PD-implicated genes with DARs between cohorts. All DARs exhibited hyperacetylation. $\mathbf{f}$ Venn diagram showing the number of PD-implicated genes with adjusted meta $P<0.05$ 
based on their adjusted $p$-value in the PW cohort) are shown in Table 1 and a complete list is provided in Supplementary Table S6.

Meta-analysis of ChIP-seq data from PW and NBB cohorts In order to perform meta-analysis of the ChIP-seq data we first harmonized the data from the two cohorts by pairing peaks with the same structural and functional genomic annotation (e.g. promoter, exon, UTR). These peaks are henceforth referred to as "common peaks". For each common peak pair, we then calculated the Fisher's meta $p$-value (metaP). A toy example of a common peak pair is shown in Fig. 4a, b.

In total, the harmonization process resulted in 52,286 pairs of common peaks. Out of these, 7288 (mapped to 2972 genes) had adjusted meta $P<0.05$ (Supplementary Table S7, Fig. 4d, f). Moreover, 300 pairs represented peaks defined as DARs in both cohorts (Fig. 4d, hypergeometric $p$-value $=5.29 \times 10^{-37}$ ). These 300 common peak pairs, mapped to 197 genes, are henceforth referred to as "replicated DARs". Nearly all (295/300) replicated DARs were hyperacetylated in both cohorts and only a single DAR, mapped to the PTPRH gene, was hypoacetylated. Genes annotated to the top replicated DARs, ranked based on their adjusted metaP, are provided in Table 2.

Table 1 Top replicated genes between PW and NBB cohorts

\begin{tabular}{lllll}
\hline GeneSymbol & log2(FC)_PW & padj_PW & log2(FC)_NBB & padj_NBB \\
\hline \multicolumn{1}{l}{ Hypoacetylated genes } & & & \\
PTPRH & -0.81 & $9.1 \mathrm{E}-03$ & -0.94 & 0.03 \\
JUP & -0.43 & 0.05 & -0.44 & 0.02 \\
Hyperacetylated genes & & & \\
DLG2 & 0.68 & $9.8 \mathrm{E}-04$ & 0.77 & $2.0 \mathrm{E}-03$ \\
TNRC6B & 0.74 & $9.8 \mathrm{E}-04$ & 0.9 & 0.03 \\
SIK3 & 0.66 & $3.2 \mathrm{E}-03$ & 0.52 & 0.01 \\
SGK1 & 0.65 & $3.2 \mathrm{E}-03$ & 0.72 & 0.01 \\
PPP2R2B & 1.3 & $3.5 \mathrm{E}-03$ & 0.7 & $8.0 \mathrm{E}-03$ \\
FCHSD2 & 0.83 & $3.5 \mathrm{E}-03$ & 1 & 0.01 \\
CTNND2 & 0.52 & $3.5 \mathrm{E}-03$ & 0.63 & 0.01 \\
PTK2 & 0.71 & $3.5 \mathrm{E}-03$ & 0.55 & 0.02 \\
SLCO1A2 & 0.58 & $3.5 \mathrm{E}-03$ & 0.68 & 0.02 \\
LPAR1 & 0.99 & $3.5 \mathrm{E}-03$ & 0.55 & 0.04 \\
FRMD4B & 0.64 & $3.5 \mathrm{E}-03$ & 0.53 & 0.04 \\
RNF216 & 0.6 & $5.1 \mathrm{E}-03$ & 0.59 & $2.3 \mathrm{E}-03$ \\
TMEM132B & 0.88 & $5.1 \mathrm{E}-03$ & 0.59 & 0.02 \\
PIP4K2A & 0.57 & $5.1 \mathrm{E}-03$ & 0.52 & 0.03 \\
MAP7 & 0.55 & $5.9 \mathrm{E}-03$ & 0.52 & 0.04 \\
PDE4B & 0.59 & $6.5 \mathrm{E}-03$ & 0.67 & $2.4 \mathrm{E}-03$ \\
FRMD5 & 0.55 & $6.5 \mathrm{E}-03$ & 0.67 & 0.01 \\
\hline
\end{tabular}

\section{Altered H3K27 acetylation has a predilection for genes with an established link to PD}

We next wondered whether genes with an established involvement in PD are over-represented among DARharboring genes. To assess this, we compiled a list of genes that fulfilled at least one of the following criteria: (a) are associated with PD based on the most recent and largest GWAS meta-analysis [4], (b) are implicated in monogenic PD [59, 60], or (c) encode proteins with a central role in PD-related neuropathology [2, 61, 62]. Among the 92 genes meeting these criteria (Supplementary Table S8), 83 could be assessed in ChIP-seq data from both cohorts and are henceforth referred to as "PD-implicated genes". Out of these, six genes: $D L G 2$, MAP 4 K4, CRHR1, MBNL2, SH3GL2 and APP, harbored DARs in both cohorts (hypergeometric $p$-value $=$ $2.9 \times 10^{-3}$, Fig. 4e). In addition, 24/83 PD-implicated genes had adjusted metaP $<0.05$ (hypergeometric $p$ value $=6.9 \times 10^{-3}$, Supplementary Table S8, Fig. 4f). These included genes with key-roles in the pathogenesis of idiopathic and/or monogenic PD: SNCA, MAPT, APP, $P R K N$ and $P A R K 7$. Interestingly, the significantly hyperacetylated peak in $S N C A$ was in an enhancer region of the gene previously shown to be affected by both genetic variation [63] and drug exposure associated with PD [64] (Fig. 5a).

\section{H3K27 hyperacetylated regions are associated with an increased ratio of $\mathrm{p} 300$ binding sites relative to non- SIRT1 HDAC binding sites}

Our immunoblot analyses suggested that H3K27 hyperacetylation may be accompanied by decreased sirtuin activity, primarily SIRT1. It is well established that SIRT1 can also deacetylate and thus suppresses the activity of the histone acetyl transferase p300 [65], the primary mediator of H3K27 acetylation [66]. Thus, we reasoned that if H3K27 hyperacetylation in PD is indeed mediated by decreased SIRT1 activity, it should be associated with regions exhibiting increased binding of p300 and decreased binding of non-SIRT1 HDACs. To test this, we integrated our data with the relevant ChIP-seq data derived from the ENCODE project [23] (see Methods). Specifically, we quantified the number of p300 bound regions (p300_binding) or non-SIRT1 HDAC bound regions (HDAC_binding) from ENCODE that overlap with H3K27ac peaks in our data and assessed the association of the two measures with H3K27 hyperacetylation level of each region. In both cohorts, the ratio of p300 binding sites over non-SIRT1 HDAC binding sites was significantly higher in regions overlapping with hyperacetylated DARs compared to non-DARs (Fig. 5c) . Notably, this was also true when restricting the analyses to the PD-implicated genes (Fig. 5d). Linear model analysis showed positive association of $\mathrm{H} 3 \mathrm{~K} 27$ hyperacetylation 
Table 2 Genes annotated to the top replicated DARs

\begin{tabular}{|c|c|c|c|c|c|c|c|c|}
\hline Symbol & $\begin{array}{l}\text { Region } \\
\text { Annotation }\end{array}$ & $\mathrm{CHR}$ & $\begin{array}{l}\text { Region } \\
\text { Location }\end{array}$ & $\begin{array}{l}\text { Peak } \\
\text { Location (PW, NBB) }\end{array}$ & MetaP & $\begin{array}{l}\text { LFC } \\
\text { PW, NBB }\end{array}$ & $\begin{array}{l}\text { PeakName } \\
\text { PW, NBB }\end{array}$ & $\begin{array}{l}\text { Proportion } \\
\text { Significant }\end{array}$ \\
\hline \multicolumn{9}{|c|}{ Common hypoacetylated regions } \\
\hline PTPRH & introns & 19 & $\begin{array}{l}55,718,568-55,720 \\
750\end{array}$ & $\begin{array}{l}55,720,288-55,722,414,55,717,912-55 \\
719,103\end{array}$ & $\begin{array}{l}1.7 \mathrm{E}- \\
07\end{array}$ & $\begin{array}{l}-0.81 \\
-0.94\end{array}$ & $\begin{array}{l}\text { Peak_20188, Peak_ } \\
15767\end{array}$ & $3^{P} / 4(0.8)$ \\
\hline$J U P$ & introns & 17 & $\begin{array}{l}39,681,526-39,923 \\
630\end{array}$ & $\begin{array}{l}39,805,764-39,808,133,39,807,092-39 \\
808,006\end{array}$ & $\begin{array}{l}1.1 \mathrm{E}- \\
05\end{array}$ & $\begin{array}{l}-0.43,- \\
0.99\end{array}$ & $\begin{array}{l}\text { Peak_53983, Peak_ } \\
81536\end{array}$ & $5^{P} / 10^{P}(C$ \\
\hline
\end{tabular}

Common hyperacetylated regions (top 15)

\begin{tabular}{|c|c|c|c|c|c|c|c|c|}
\hline$D L G 2^{a}$ & exons & 11 & $\begin{array}{l}83,874,504-83,874 \\
554\end{array}$ & $\begin{array}{l}83,873,551-83,879,844,83,873,666-83, \\
877,544\end{array}$ & $\begin{array}{l}7.7 \mathrm{E}- \\
12\end{array}$ & $0.68,1.05$ & $\begin{array}{l}\text { Peak_21808, Peak_ } \\
40741\end{array}$ & $\begin{array}{l}30^{p /} \\
49^{P}(0.6)\end{array}$ \\
\hline KIT & exons & 4 & $\begin{array}{l}55,604,595-55,606 \\
881\end{array}$ & $\begin{array}{l}55,606,747-55,609,274,55,606,782-55 \\
609,553\end{array}$ & $\begin{array}{l}8.1 \mathrm{E}- \\
11\end{array}$ & $0.63,1.26$ & $\begin{array}{l}\text { Peak_21777, Peak_ } \\
37103\end{array}$ & $2 / 3^{\mathrm{P}}(0.7)$ \\
\hline $\begin{array}{l}\text { CNTN } \\
\text { AP2 }\end{array}$ & exons & 7 & $\begin{array}{l}147,336,198-147 \\
336,398\end{array}$ & $\begin{array}{l}147,333,973-147,338,260,147,333 \\
944-147,337,213\end{array}$ & $\begin{array}{l}6.2 \mathrm{E}- \\
09\end{array}$ & $0.51,1.07$ & $\begin{array}{l}\text { Peak_44999, Peak_ } \\
47588\end{array}$ & $11 / 18^{p}(0.6$ \\
\hline NF216 & introns & 7 & $\begin{array}{l}5,681,008-5,692, \\
043\end{array}$ & $\begin{array}{l}5,679,685-5,685,127,5,688,370-5,705 \\
729\end{array}$ & $\begin{array}{l}1.2 \mathrm{E}- \\
08\end{array}$ & $0.6,0.53$ & $\begin{array}{l}\text { Peak_38631, Peak_ } \\
12618\end{array}$ & $11^{\mathrm{p}} / 16(0.7)$ \\
\hline FRMD5 & introns & 15 & $\begin{array}{l}44,216,508-44,393 \\
656\end{array}$ & $\begin{array}{l}44,260,324-44,263,821,44,244,249-44, \\
247,825\end{array}$ & $\begin{array}{l}1.6 \mathrm{E}- \\
08\end{array}$ & $0.68,0.67$ & $\begin{array}{l}\text { Peak_18018, Peak_ } \\
18744\end{array}$ & $\begin{array}{l}12^{\mathrm{P}} / \\
17^{\mathrm{P}}(0.7)\end{array}$ \\
\hline $\begin{array}{l}\text { SOX2- } \\
\text { OT }\end{array}$ & introns & 3 & $\begin{array}{l}181,328,718-181 \\
417,385\end{array}$ & $\begin{array}{l}181,358,880-181,360,150,181,341 \\
154-181,348,014\end{array}$ & $\begin{array}{l}1.7 \mathrm{E}- \\
08\end{array}$ & $0.55,0.91$ & $\begin{array}{l}\text { Peak_43667, Peak_ } \\
31549\end{array}$ & $9 / 12(0.8)$ \\
\hline MAGI2 & 1 to5kb & 7 & $\begin{array}{l}78,120,338-78,124 \\
337\end{array}$ & $\begin{array}{l}78,121,257-78,128,843,78,115,190-78, \\
121,170\end{array}$ & $\begin{array}{l}2.2 \mathrm{E}- \\
08\end{array}$ & $0.4,0.93$ & $\begin{array}{l}\text { Peak_18816, Peak_ } \\
24042\end{array}$ & $9^{P} / 23(0.4)$ \\
\hline$P P P 2 R 2 B$ & introns & 5 & $\begin{array}{l}146,080,706-146 \\
460,621\end{array}$ & $\begin{array}{l}535-146,384,203,146,459 \\
469,815\end{array}$ & $\begin{array}{l}2.8 \mathrm{E}- \\
08\end{array}$ & $1.3,0.52$ & $\begin{array}{l}35972 \\
7645\end{array}$ & $\begin{array}{l}17^{P} / \\
27^{P}(0.6)\end{array}$ \\
\hline KCNH1 & introns & 1 & $\begin{array}{l}210,948,887-210 \\
970,849\end{array}$ & $\begin{array}{l}210,966,438-210,969,215,210,946 \\
491-210,950,181\end{array}$ & $\begin{array}{l}3.8 \mathrm{E}- \\
08\end{array}$ & $0.55,1.0$ & $\begin{array}{l}\text { Peak_51838, Peak_ } \\
38212\end{array}$ & $9 / 18^{p}(0.5)$ \\
\hline $\mathrm{CDH} 1$ & introns & 16 & $\begin{array}{l}68,835,797-68,842 \\
326\end{array}$ & $\begin{array}{l}68,840,159-68,841,130,68,782,065-68, \\
837,136\end{array}$ & $\begin{array}{l}4.8 \mathrm{E}- \\
08\end{array}$ & $0.84,0.37$ & $\begin{array}{l}\text { Peak_66179, Peak_ } \\
3389\end{array}$ & $5 / 7^{\mathrm{P}}(0.7)$ \\
\hline $\begin{array}{l}\text { MAP } 4 \\
K 4^{\mathrm{a}}\end{array}$ & introns & 2 & $\begin{array}{l}102,315,001-102 \\
407,181\end{array}$ & $\begin{array}{l}963-102,334,475,102,367 \\
2,375,949\end{array}$ & $\begin{array}{l}5.2 \mathrm{E}- \\
08\end{array}$ & $0.54,0.62$ & $\begin{array}{l}\text { Peak_30093, Peak_ } \\
9009\end{array}$ & $\begin{array}{l}10^{\mathrm{P}} / \\
24^{\mathrm{P}}(0.4)\end{array}$ \\
\hline $\begin{array}{l}\text { METT } \\
\text { L24 }\end{array}$ & introns & 6 & $\begin{array}{l}110,644,076-110 \\
729,536\end{array}$ & $\begin{array}{l}110,656,829-110,661,962,110,685 \\
537-110,688,887\end{array}$ & $\begin{array}{l}5.4 \mathrm{E}- \\
08\end{array}$ & $0.48,0.97$ & $\begin{array}{l}\text { Peak_22739, Peak_ } \\
27591\end{array}$ & $8 / 13^{P}(0.6)$ \\
\hline PIP4K2A & introns & 10 & $\begin{array}{l}22,898,647-23,003 \\
111\end{array}$ & $\begin{array}{l}22,934,570-22,952,399,22,957,818-22, \\
962,517\end{array}$ & $\begin{array}{l}5.6 \mathrm{E}- \\
08\end{array}$ & $0.58,0.52$ & $\begin{array}{l}\text { Peak_22480, Peak_ } \\
27954\end{array}$ & $9^{P} / 18^{P}(0.5)$ \\
\hline SIK3 & introns & 11 & $\begin{array}{l}116,827,781-116 \\
968,858\end{array}$ & $\begin{array}{l}116,825,819-116,835,556,116,846 \\
923-116,884,577\end{array}$ & $\begin{array}{l}5.8 \mathrm{E}- \\
08\end{array}$ & $0.6,0.49$ & $\begin{array}{l}\text { Peak_21504, Peak_ } \\
11226\end{array}$ & $\begin{array}{l}13^{\mathrm{P} /} \\
16^{\mathrm{P}}(0.8)\end{array}$ \\
\hline DOCK10 & introns & 2 & $\begin{array}{l}225,796,386-225 \\
906,968\end{array}$ & $\begin{array}{l}225,798,681-225,800,264,225,845 \\
772-225,849,871\end{array}$ & $\begin{array}{l}8.1 \mathrm{E}- \\
08\end{array}$ & $0.61,0.77$ & $\begin{array}{l}\text { Peak_94440, Peak_ } \\
22866\end{array}$ & $6 / 10^{P}(0.6)$ \\
\hline \multicolumn{9}{|c|}{ gions (additional GWAS hits) } \\
\hline$C R H R 1^{\mathrm{a}}$ & introns & 17 & $\begin{array}{l}43,707,525-43,750 \\
192\end{array}$ & $\begin{array}{l}43,726,544-43,728,643,43,741,886-43, \\
747,152\end{array}$ & $\begin{array}{l}2.0 \mathrm{E}- \\
07\end{array}$ & $0.43,0.83$ & $\begin{array}{l}\text { Peak_74390, Peak_ } \\
30944\end{array}$ & $6 / 14^{P}(0.4)$ \\
\hline$M B N L 2^{\mathrm{a}}$ & introns & 13 & $\begin{array}{l}97,873,814-97,927 \\
885\end{array}$ & $\begin{array}{l}97,919,075-97,922,481,97,906,667-97, \\
916,758\end{array}$ & $\begin{array}{l}1.2 \mathrm{E}- \\
05\end{array}$ & $0.44,0.44$ & $\begin{array}{l}\text { Peak_29656, Peak_ } \\
16279\end{array}$ & $\begin{array}{l}14^{\mathrm{P}} / \\
22^{\mathrm{P}}(0.6)\end{array}$ \\
\hline
\end{tabular}

For hyperacetylated regions, the top 15 genes with DARs common to the two cohorts and common DARs in genes previously associated with PD are shown. The genes were ranked based on Fisher's meta $p$-value. The full list of genes is provided in Supplementary Table 56

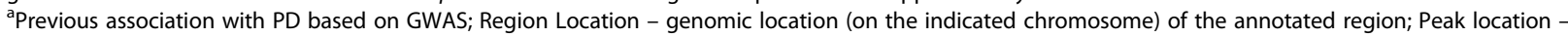
genomic location of the relevant peak from each cohort (upper - PW, lower - NBB); LFC - log fold change compared to controls; Peak Name - the peak identifier in each cohort; Proportion significance - DARs/total common regions annotated to the gene; ${ }^{{ }^{p}}$ - peaks annotated to promoter region of the gene

with p300_binding (PW: $\beta=0.02, p<2 \times 10^{-15}$; NBB: $\beta=0.09, \quad p<10^{-26}$ ) and negative association with HDAC_binding (PW: $\beta=-0.07, \mathrm{p}<10^{-26}$; NBB: $\beta=-$ $\left.0.14, \mathrm{p}<10^{-26}\right)$. Similar results were obtained when peaks were collapsed to a gene level (Supplementary Table S9).

\section{Differential H3K27 acetylation is associated with effective gene length}

We noted that many of the genes with DARs, including PD-implicated genes, are among the longest in the genome. We reasoned that this may be because longer genes have higher histone binding capacity and therefore 


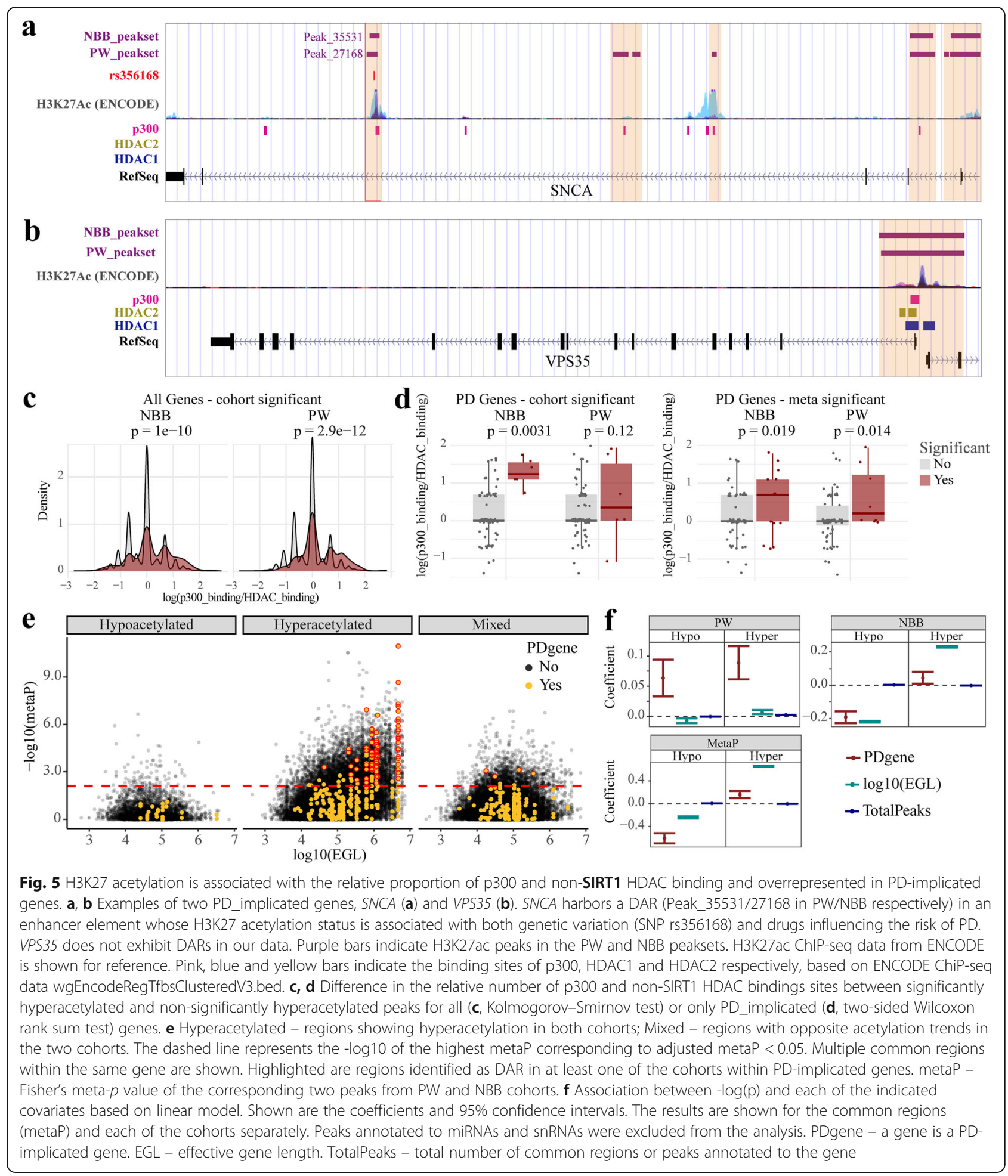

are more likely to show a differential signal in the event of a generalized, unspecific H3K27 hyperacetylation. To test this hypothesis and ensure that the overrepresentation of PD-implicated genes among DARharboring genes is not driven by longer gene length, we used linear models to assess whether H3K27ac, defined as $-\log 10$ (metaP), is associated with PD-implicated genes while accounting for the effective gene length (EGL), defined as the total length of all regions annotated to a gene (see Methods). The total number of common regions in a gene was included as a covariate in the model. For hyperacetylated regions, this analysis indicated a 
significant positive association between altered acetylation and PD-implicated genes $\left(p=1.0 \times 10^{-7}\right)$ as well as EGL $\left(p<2.0 \times 10^{-26}\right)$. For the hypoacetylated regions, however, the analysis indicated a significant negative association with both PD-implicated genes $(\mathrm{p}<2.0 \times$ $\left.10^{-26}\right)$ and EGL $\left(\mathrm{p}<2.0 \times 10^{-26}\right)$. Analysis of each cohort independently corroborated these findings, although the negative association with PD-implicated genes for the hypoacetylated regions was only significant in the NBB cohort (Fig. 5b, Supplementary Table S10).

\section{DARs differ in their GC content and are enriched in multiple transcription factor motifs}

In both cohorts, the hyperacetylated DARs exhibited low GC content compared to non-differentially acetylated regions, matched for region length (NBB: $44 \%$ vs $51 \%$; PW: $46 \%$ vs. $50 \%$, DARs vs. non-DARs, respectively, Supplementary Fig. S8). The opposite phenomenon was observed for the hypoacetylated DARs (NBB: 61\% vs 51\%; PW: $55 \%$ vs. $50 \%$, DARs vs. non-DARs, respectively, Supplementary Fig. S8). Similar results were obtained when comparison was performed only for peaks annotated to either exonic or intronic regions. Motif enrichment analysis of the hyper- and hypoacetylated DARs indicated enrichment in multiple TFBMs. For the hyperacetylated DARs, significant enrichment was found for 71 and 94. TFBMs, in the PW and NBB cohorts respectively, with 64 TFBMs overlapping between the two cohorts. For the hypoacetylated DARs, significant enrichment was found for 3 and 49 TFBMs, in the PW and NBB cohorts respectively, two of which were enriched in both (Supplementary Table S11).

\section{Promoter H3K27 acetylation is decoupled from gene expression in PD}

While it is well accepted that modifications of H3K27ac are predictive of gene expression levels [67], it was previously shown that widespread histone hyperacetylation induced by potent HDAC inhibition does not lead to major changes in gene expression [68,69]. To investigate whether a similar decoupling occurs in the $\mathrm{PD}$ brain, we analyzed RNA sequencing (RNA-seq) data from the PFC of the same individuals included in the ChIP-seq analysis (Supplementary Table 1, Supplementary Methods). For each gene, we then calculated the Pearson's correlation between the level of its expression and promoter H3K27ac across controls or individuals with PD.

In the control group from both cohorts, most genes exhibited the expected positive correlation between promoter H3K27ac and expression. In the PD group, however, the distribution of correlations was centered closer to 0 , regardless of the absolute correlation threshold chosen (Fig. 6a-e). The deviation between the two groups progressively increased as only genes with higher correlation between promoter H3K27ac and expression were included in the analysis (Fig. 6b-e). Importantly, similar results were obtained based on RLE-normalized ChIP-seq data (Supplementary Fig. S9), indicating that the observed decreased correlation between H3K27ac and transcription levels in PD was not driven by the normalization method. These results suggest that the coupling between promoter H3K27 acetylation state and proximal gene transcription is impaired in the PFC of individuals with PD.

Functional enrichment analysis based on the decoupling level of the genes, indicated that nucleus-encoded mitochondrial genes are the most decoupled genes in both cohorts (Supplementary Table S12). Moreover, closer examination of these genes revealed that most of them exhibit negative correlation between the expression level and promoter H3K27 acetylation (Fig. 6f, Supplementary Table S12).

\section{Discussion}

\section{Aberrant H3K27ac occurs genome-wide in the PD brain}

We report evidence of increased histone acetylation in the PFC of individuals with PD with the strongest change observed for H3K27, a marker of active promoters and enhancers with a fundamental role in regulating gene expression [70, 71]. H3K27 hyperacetylation in PD is supported by multiple levels of evidence in our study: (a) immunoblot analyses showed a highly significant increase in the acetylated fraction of H3K27 across three brain regions in PD. Importantly, we show that this is not driven by underlying differences in cell composition of the samples and is unlikely to be induced by anti-Parkinson medication. (b) ChIP-seq analysis, replicated in two independent cohorts, showed that the total number of peaks, percentage of unique peaks, genome coverage and the total number of RiPs are all increased in the PD samples. The analysis further revealed that increased H3K27ac occupancy has a genome-wide distribution. (c) We found a strong association between differential H3K27 acetylation and the effective length of the targeted gene. This association was positive for hyperacetylated regions and negative for hypoacetylated regions, in line with a hyperacetylation-predisposing cellular environment in PD.

\section{H3K27 hyperacetylation may be induced by altered sirtuin activity}

We hypothesized that H3K27 hyperacetylation in PD may be mediated by altered sirtuin activity, due to a decreased $\mathrm{NAD}^{+} / \mathrm{NADH}$ ratio $[39,42,72]$ resulting from complex-I deficiency, a pathological hallmark of the PD brain [38, 73]. Hyperacetylation of several histone sites, including H3K27, was indeed observed in animal and 


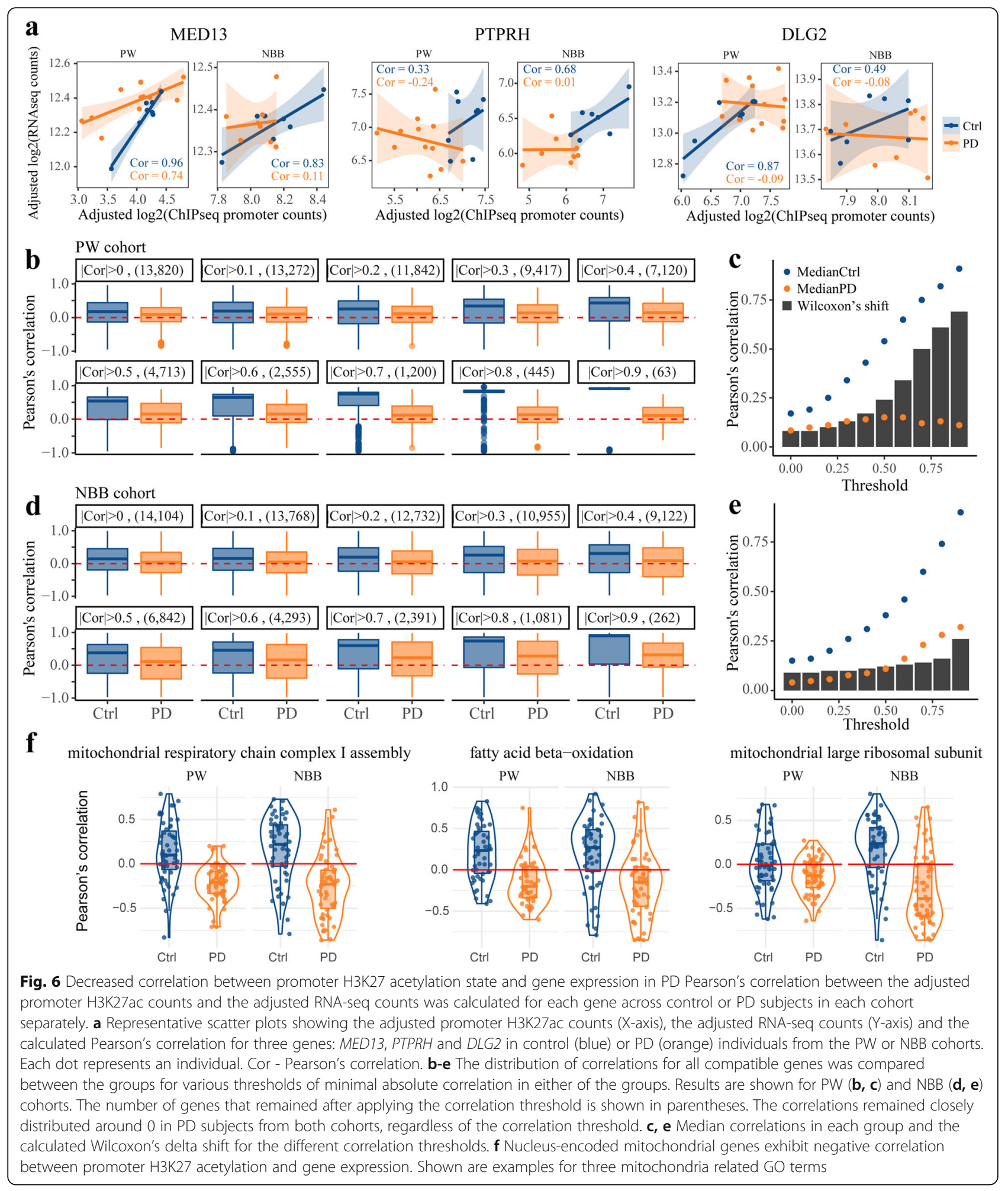

cell studies, following chemical complex-I inhibition with PD-associated neurotoxins [10, 74]. In line with our hypothesis, we observed increased levels of SIRT1 and SIRT3 proteins in PD samples and no change in SIRT2 quantity, or in the acetylation state of its substrate, $\alpha$ - tubulin. This is consistent with the notion that SIRT1 and SIRT3, but not SIRT2 activity are susceptible to changes in physiological $\mathrm{NAD}^{+}$concentrations [72]. In line with our findings, reduced sirtuin activity accompanied by increase in their protein levels was reported in 
iPSC-derived dopaminergic cells carrying a $L R R K 2 \mathrm{mu}-$ tation [75]. Moreover, complex-I deficiency, decreased $\mathrm{NAD}^{+} / \mathrm{NADH}$ ratio, decreased sirtuin activity (accompanied by increased protein levels), and increased histone acetylation have all been shown to occur with aging $[5,41,44,46,47]$, which is the strongest known risk factor for PD.

The putative role of SIRT1 in mediating H3K27 hyperacetylation in PD is further supported by the observation that hyperacetylation is more pronounced among regions with high $\mathrm{p} 300 /$ non-SIRT1 HDAC binding site ratio. This is because decreased SIRT1 activity increases p300-mediated H3K27 acetylation, and can be compensated by other HDACs. Namely, H3K27 hyperacetylation induced by decreased SIRT1 activity is expected be more pronounced in regions exhibiting multiple p300 binding sites and fewer non-SIRT1 HDAC binding sites. While our findings strongly suggest the sirtuins may be involved in H3K27 hyperacetylation in PD, further studies are required to validate this effect, and to establish whether this involvement is direct (i.e. by deacetylation of H3K27) or indirect (e.g., via reduced deacetylation and thereby activation of $\mathrm{p} 300$ ).

\section{H3K27 hyperacetylation is over-represented in PD- implicated genes}

While H3K27 hyperacetylation appears to be a genomewide phenomenon in the PD brain, it also shows a highly significant predilection for genes implicated in PD. Meta-analysis of our two cohorts identified H3K27 hyperacetylated regions in 24/83 genes linked to idiopathic and/or monogenic forms of PD (Supplementary Table S8). These include 21 genes associated with PD by GWAS [4], genes encoding proteins involved in PD pathology (SNCA, MAPT, APP) [2, 61, 62] and genes in which mutations cause Mendelian PD (SNCA, PRKN, PARK7) [76, 77]. In addition, H3K27 hyperacetylation was observed in genes associated with more complex forms of parkinsonism and/or vulnerability to PDrelated pathology such as FBOX7 [78] and POLG [59].

Several mechanisms may underlie the observed overrepresentation of PD-implicated genes. First, it is plausible that H3K27 acetylation at promoter and/or enhancer regions of these genes is modulated by genetic variation and/or environmental factors associated with PD. For instance, the H3K27 acetylation state of the SNCA enhancer region, significantly hyperacetylated in our data, was shown to be affected by both genetic variation [63] and drug exposure associated with PD [64]. Second, it is likely that pathogenic processes involved in PD induce the expression of genes implicated in the disease. For example, the expression levels of PRKN and $P A R K 7$ increase in response to oxidative stress [79-82]. Since, the regulation of transcription often involves histone acetylation, impairment of the deacetylation machinery, would over time lead to excessive H3K27 acetylation of these genes. Finally, by relaxing chromatin structure, H3K27 hyperacetylation in genomic regions associated with PD, predisposes these areas to somatic mutations, including genomic rearrangements [83]. As previously suggested [84], this could act synergistically with other predisposing factors, such as age and oxidative injury, eventually leading to PD-specific pathology.

\section{H3K27 hypoacetylation in the PTPRH gene may contribute to PD pathophysiology}

Considering the general H3K27 hyperacetylation in the PD brain, the robust hypoacetylation of H3K27 in the PTPRH gene, encoding protein tyrosine phosphatase receptor type $\mathrm{H}$, is of particular interest. While little is known about the physiological function of PTPRH, it was previously found to be enriched in loss-of-function mutations in PD [57]. Furthermore, it was shown that RNAi-mediated downregulation of the PTPRH homolog in Drosophila enhances $\alpha$-synuclein neurotoxicity [57]. Our finding of selective H3K27 hypoacetylation of the PTPRH region in PD, corroborates the hypothesis that decreased function of this gene may contribute to the pathophysiology of PD. Additional studies are required to establish the physiological function of PTPRH and its involvement in the pathophysiology of PD.

\section{Differential H3K27ac is associated with GC content}

The observation that hyperacetylated DARs exhibit low GC content, whereas hypoacetylated DARS exhibit high GC content, is intriguing. While the reason behind this phenomenon is unclear, the existence of enhancers with high and low GC content was previously described, linking low GC content-enhancers to the immune response [85]. Additional studies are required to understand the mechanism underlying the selectivity of differential H3K27ac towards different sequence composition and its link to PD.

\section{Decoupling between promoter H3K27 acetylation and gene expression in the PD brain}

The attenuated correlation between promoter H3K27 acetylation and gene expression is intriguing, since it indicates that a fundamental mechanism of gene regulation is defective in the PD brain. A plausible explanation for this phenomenon is a global hyperacetylation of enhancer regions, compromising the regulation of gene expression. Indeed, in both cohorts, about half of the identified H3K27ac peaks as well as DARs were annotated to non-coding regions. Furthermore, since histone acetylation is tightly linked to the metabolic state of the cell [72], this decoupling suggests that cellular responses to metabolic stress may be severely compromised in PD. 
This putative compromised response to metabolic stress is further supported by the finding that, in both cohorts, the highest degree of decoupling is observed among nuclear-encoded mitochondrial genes. Moreover, the negative correlation between H3K27 acetylation and gene expression of these genes supports the involvement of sirtuins, in particular SIRT1, in the pathophysiology of PD. This is because in addition to histones, SIRT1 deacetylates and activates PGC-1 $\alpha$, the key regulator of mitochondrial biogenesis [42, 72, 86]. Thus, decreased SIRT1 activity would simultaneously lead to increased histone acetylation and reduced expression of nuclearencoded mitochondrial genes, resulting in negative correlation between their expression level and promoter H3K27 acetylation state. Moreover, decreased expression of mitochondrial genes would initiate a vicious circle by decreasing $\mathrm{NAD}^{+} / \mathrm{NADH}$ ratio and further reducing SIRT1 activity.

\section{Conclusions}

We show that H3K27 acetylation, a fundamental mechanism modulating gene expression, is severely dysregulated in the PFC of individuals with idiopathic PD. The highly significant concordance between the two cohorts and the clear predilection for genes strongly linked to PD, validate our findings and suggest that epigenetic dysregulation plays an important role in the pathogenesis of the disease. Importantly, since histone acetylation and NAD metabolism can be pharmaceutically modulated, our results open the possibility that agents targeting these biological processes may have therapeutic potential for PD and should be further explored.

\begin{abstract}
Abbreviations
ChIP-seq: Chromatin immunoprecipitation sequencing; Ctrl: Control; DAR: Differentially acetylated region; EGL: Effective gene length; GWAS: Genome-wide association study; HAT: Histone acetyltransferase; HDAC: Histone deacetylase; L-DOPA: Levodopa; mtDNA: Mitochondrial DNA; MSPs: Marker Site Profiles; NAD: Nicotinamide adenine dinucleotide; NBB: Netherlands Brain Bank; PD: Parkinson's disease; PFC: Prefrontal cortex; PMI: Postmortem interval; PW: Park West; RiP: Reads in peaks; RLE: Relative Log Expression; SNc: Substantia nigra pars compacta; TSS: Transcription start site; WB: Western blot; WES: Whole exome sequencing
\end{abstract}

\section{Supplementary Information}

The online version contains supplementary material available at https://doi. org/10.1186/s13024-021-00450-7.

\section{Additional file 1.}

\section{Acknowledgments}

This work is supported by grants from The Research Council of Norway (ES633272) and Bergen Research Foundation (BFS2017REK05). The cell-type specific H3K27ac ChIP-seq data were generated as part of the PsychENCODE Consortium, supported by: U01MH103392, U01MH103365, U01MH103346, U01MH103340, U01MH103339, R21MH109956, R21MH105881, R21MH105853, R21MH103877, R21MH102791, R01MH111721, R01MH110928, R01MH110927, R01MH1 10926, R01MH1 10921, R01MH110920, R01MH110905, R01MH109715, R01MH109677, R01MH105898, R01MH105898, R01MH094714, P50MH106934,
U01MH1 16488, U01MH116487, U01MH116492, U01MH116489, U01MH116438, U01MH116441, U01MH116442, R01MH114911, R01MH114899, R01MH114901, R01MH117293, R01MH117291, R01MH117292 awarded to: Schahram Akbarian (Icahn School of Medicine at Mount Sinai), Gregory Crawford (Duke University), Stella Dracheva (Icahn School of Medicine at Mount Sinai), Peggy Farnham (University of Southern California), Mark Gerstein (Yale University), Daniel Geschwind (University of California, Los Angeles), Fernando Goes (Johns Hopkins University), Thomas M. Hyde (Lieber Institute for Brain Development), Andrew Jaffe (Lieber Institute for Brain Development), James A. Knowles (University of Southern California), Chunyu Liu (SUNY Upstate Medical University), Dalila Pinto (Icahn School of Medicine at Mount Sinai), Panos Roussos (Icahn School of Medicine at Mount Sinai), Stephan Sanders (University of California, San Francisco), Nenad Sestan (Yale University), Pamela Sklar (Icahn School of Medicine at Mount Sinai), Matthew State (University of California, San Francisco), Patrick Sullivan (University of North Carolina), Flora Vaccarino (Yale University), Daniel Weinberger (Lieber Institute for Brain Development), Sherman Weissman (Yale University), Kevin White (University of Chicago), Jeremy Willsey (University of California, San Francisco), and Peter Zandi (Johns Hopkins University). We are grateful to Prof. Laurence A. Bindoff and Dr. Shreejoy Tripathy for the valuable comments and discussions.

\section{Authors' contributions}

L.T. conceived, designed and performed the ChIP-Seq and integrative computational analyses. G.T.T. designed and performed the immunoblots and cell-culture experiments. J.S. contributed to conception and implementation of the ChIP-seq and immunoblot analyses. K.H. contributed to data interpretation and critical revision of the manuscript. G.S.N. performed the preprocessing of the ChIP-seq dataset and contributed to the computational analyses. C.D. conceived, designed, supervised the immunoblots and cell-culture experiments, performed part of the immunoblot experiments, contributed to data analyses and interpretation. O.B.T. and G.A. contributed part of the tissue material, supplementary data, and critical revision of the manuscript. C.T. conceived, designed and directed the study, contributed to data analyses and interpretation and acquired funding for the study. L.T., G.T.T., C.D. and C.T. wrote the manuscript with the active input and participation of J.S. and G.S.N. All authors have read and approved the manuscript.

\section{Funding}

This work is supported by grants from The Research Council of Norway (288164, ES633272) and Bergen Research Foundation (BFS2017REK05).

\section{Availability of data and materials}

Raw counts for the ChIP-seq analyses are available in the same repository (https://github.com/ltoker/ChIPseqPD/tree/master/data). The corresponding RNA-seq raw data counts are included in the repository (https://git.app.uib. no/neuromics/cell-composition-rna-pd/tree/master/Data).

\section{Declarations}

Ethics approval and consent to participate

Informed consent was available from all individuals. Ethical permission for these studies was obtained from our regional ethics committee (REK 2017/ 2082, 2010/1700, 131.04).

\section{Consent for publication}

Not applicable.

\section{Competing interests}

The authors report no competing financial interests.

\section{Author details}

${ }^{1}$ Neuro-SysMed Center of Excellence for Clinical Research in Neurological Diseases, Department of Neurology, Haukeland University Hospital, 5021 Bergen, Norway. ${ }^{2}$ Department of Clinical Medicine, University of Bergen, $\mathrm{Pb}$ 7804, 5020 Bergen, Norway. ${ }^{3}$ The Norwegian Centre for Movement Disorders and Department of Neurology, Stavanger University Hospital, Pb 8100, 4068 Stavanger, Norway. ${ }^{4}$ Department of Mathematics and Natural Sciences, University of Stavanger, 4062 Stavanger, Norway. 
Received: 9 October 2020 Accepted: 14 April 2021 Published online: 05 May 2021

\section{References}

1. de Rijk MC, Launer L, Berger K, Breteler MM, Dartigues JF, Baldereschi M, et al. Prevalence of Parkinson's disease in Europe: a collaborative study of population-based cohorts. Neurologic diseases in the elderly research group. Neurology. 2000;54(11 Suppl 5):S21-3.

2. Dickson DW. Parkinson's disease and parkinsonism: neuropathology. Cold Spring Harb Perspect Med. 2012;2 Available from: http://www.ncbi.nlm.nih. gov/pubmed/22908195.

3. Klein C, Westenberger A. Genetics of Parkinson's disease. Cold Spring Harb Perspect Med. 2012;2:a008888.

4. Nalls MA, Blauwendraat C, Vallerga CL, Heilbron K, Bandres-Ciga S, Chang D, et al. Identification of novel risk loci, causal insights, and heritable risk for Parkinson's disease: a meta-analysis of genome-wide association studies. Lancet Neurol. 2019;18(12):1091-102. https://doi.org/10.1016/S1474-4422 (19)30320-5

5. Peleg S, Feller C, Ladurner AG, Imhof A. The metabolic impact on histone acetylation and transcription in ageing. Trends Biochem Sci. 2016;41(8):70011. https://doi.org/10.1016/j.tibs.2016.05.008.

6. Saha R, Pahan K. HATs and HDACs in neurodegeneration: a tale of disconcerted acetylation homeostasis. Cell Death Differ. 2006;13(4):539-50. https://doi.org/10.1038/sj.cdd.4401769.

7. Nativio R, Donahue G, Berson A, Lan Y, Amlie-Wolf A, Tuzer F, et al. Dysregulation of the epigenetic landscape of normal aging in Alzheimer's disease. Nat Neurosci. 2018;21(4):497-505. https://doi.org/10.1038/s41593-01 8-0101-9.

8. Marzi SJ, Leung SK, Ribarska T, Hannon E, Smith AR, Pishva E, et al. A histone acetylome-wide association study of Alzheimer's disease identifies diseaseassociated H3K27ac differences in the entorhinal cortex. Nat Neurosci. 2018; 21(11):1618-27. https://doi.org/10.1038/s41593-018-0253-7.

9. Harrison IF, Smith AD, Dexter DT. Pathological histone acetylation in Parkinson's disease: Neuroprotection and inhibition of microglial activation through SIRT 2 inhibition. Neurosci Lett. 2018;666:48-57. https://doi.org/10.1 016/j.neulet.2017.12.037.

10. Park G, Tan J, Garcia G, Kang Y, Salvesen G, Zhang Z. Regulation of histone acetylation by autophagy in Parkinson disease. J Biol Chem. 2016;291(7): 3531-40. https://doi.org/10.1074/jbc.M115.675488.

11. Gebremedhin $\mathrm{KG}$, Rademacher DJ. Histone $\mathrm{H3}$ acetylation in the postmortem Parkinson's disease primary motor cortex. Neurosci Lett. 2016; 627:121-5. https://doi.org/10.1016/j.neulet.2016.05.060.

12. Alves G, Muller B, Herlofson K, HogenEsch I, Telstad W, Aarsland D, et al. Incidence of Parkinson's disease in Norway: the Norwegian ParkWest study. J Neurol Neurosurg Psychiatry. 2009;80(8):851-7. https://doi.org/10.1136/ jnnp.2008.168211.

13. Gaare JJ, Nido GS, Sztromwasser P, Knappskog PM, Dahl O, Lund-Johansen $M$, et al. Rare genetic variation in mitochondrial pathways influences the risk for Parkinson's disease: mitochondrial pathways in PD. Mov Disord. 2018; 33(10):1591-600. https://doi.org/10.1002/mds.64.

14. Gelb DJ, Oliver E, Gilman S. Diagnostic criteria for Parkinson disease. Arch Neurol. 1999;56(1):33-9. https://doi.org/10.1001/archneur.56.1.33.

15. Ward CD, Gibb WR. Research diagnostic criteria for Parkinson's disease. Adv Neurol. 1990;53:245-9.

16. Bolger AM, Lohse M, Usadel B. Trimmomatic: a flexible trimmer for Illumina sequence data. Bioinformatics. 2014;30(15):2114-20. https://doi.org/10.1093/ bioinformatics/btu170

17. Li H, Durbin R. Fast and accurate long-read alignment with burrowswheeler transform. Bioinformatics. 2010;26(5):589-95. https://doi.org/10.1 093/bioinformatics/btp698.

18. Liao Y, Smyth GK, Shi W. featureCounts: an efficient general purpose program for assigning sequence reads to genomic features. Bioinforma Oxf Engl. 2014;30(7):923-30. https://doi.org/10.1093/bioinformatics/btt656.

19. Girdhar K, Hoffman GE, Jiang Y, Brown L, Kundakovic M, Hauberg ME, et al Cell-specific histone modification maps in the human frontal lobe link schizophrenia risk to the neuronal epigenome. Nat Neurosci. 2018;21(8): 1126-36. https://doi.org/10.1038/s41593-018-0187-0.

20. Love Ml, Huber W, Anders S. Moderated estimation of fold change and dispersion for RNA-seq data with DESeq2. Genome Biol. 2014;15(12):550. https://doi.org/10.1186/s13059-014-0550-8.
21. Cavalcante RG, Sartor MA. annotatr: genomic regions in context. Bioinforma Oxf Engl. 2017;33(15):2381-3. https://doi.org/10.1093/bioinformatics/btx183.

22. Mancarci BO, Toker L, Tripathy SJ, Li B, Rocco B, Sibille E, et al. CrossLaboratory Analysis of Brain Cell Type Transcriptomes with Applications to Interpretation of Bulk Tissue Data. eNeuro. 2017;4(6):ENEURO.0212-17.2017. https://doi.org/10.1523/ENEURO.0212-17.2017.

23. ENCODE Project Consortium. The ENCODE (ENCyclopedia of DNA elements) project. Science. 2004;306(5696):636-40. https://doi.org/10.1126/science.11 05136.

24. Lawrence $M$, Huber W, Pagès $H$, Aboyoun P, Carlson M, Gentleman R, et al. Software for computing and annotating genomic ranges. PLoS Comput Biol. 2013;9(8):e1003118. https://doi.org/10.1371/journal.pcbi.1 003118.

25. McLeay RC, Bailey TL. Motif enrichment analysis: a unified framework and an evaluation on ChIP data. BMC Bioinformatics. 2010;11(1):165. https://doi. org/10.1186/1471-2105-11-165.

26. Bailey TL, Boden M, Buske FA, Frith M, Grant CE, Clementi L, et al. MEME SUITE: tools for motif discovery and searching. Nucleic Acids Res. 2009; 37(Web Server):W202-8. https://doi.org/10.1093/nar/gkp335.

27. Kulakovskiy IV, Vorontsov IE, Yevshin IS, Sharipov RN, Fedorova AD, Rumynskiy El, et al. HOCOMOCO: towards a complete collection of transcription factor binding models for human and mouse via large-scale ChIP-Seq analysis. Nucleic Acids Res. 2018;46(D1):D252-9. https://doi.org/1 0.1093/nar/gkx1106

28. Nido GS, Dick F, Toker L, Petersen K, Alves G, Tysnes O-B, et al. Common gene expression signatures in Parkinson's disease are driven by changes in cell composition. Acta Neuropathol Commun. 2020;8(1):55. https://doi.org/1 0.1186/s40478-020-00932-7.

29. Toker L, Mancarci BO, Tripathy S, Pavlidis P. Transcriptomic evidence for alterations in astrocytes and Parvalbumin interneurons in subjects with bipolar disorder and schizophrenia. Biol Psychiatry. 2018;84(11):787-96. https://doi.org/10.1016/j.biopsych.2018.07.010.

30. Mancarci O. ErmineR (R package). Available from: https://github.com/Pa vlidisLab/ermineR. Accessed June 2020.

31. Gillis J, Mistry M, Pavlidis P. Gene function analysis in complex data sets using ErmineJ. Nat Protoc. 2010;5(6):1148-59. https://doi.org/10.1038/nprot.2 010.78 .

32. Ashburner M, Ball CA, Blake JA, Botstein D, Butler H, Cherry JM, et al. Gene ontology: tool for the unification of biology. The Gene Ontology Consortium. Nat Genet. 2000;25(1):25-9. https://doi.org/10.1038/75556.

33. Taylor AE, Saint-Cyr JA, Lang AE. Frontal lobe dysfunction in Parkinson's disease. The cortical focus of neostriatal outflow. Brain J Neurol. 1986;109(Pt 5):845-83. https://doi.org/10.1093/brain/109.5.845.

34. Yau Y, Zeighami Y, Baker TE, Larcher K, Vainik U, Dadar M, et al. Network connectivity determines cortical thinning in early Parkinson's disease progression. Nat Commun. 2018;9(1):12. https://doi.org/10.1038/s41467-017-02416-0.

35. Prell T. Structural and Functional Brain Patterns of Non-Motor Syndromes in Parkinson's Disease. Front Neurol. 2018;9 [cited 2020 Jul 14] Available from: https://www.frontiersin.org/articles/10.3389/fneur.2018.00138/full.

36. Seidel K, Bouzrou M, Heidemann N, Krüger R, Schöls L, den Dunnen WFA, et al. Involvement of the cerebellum in Parkinson disease and dementia with Lewy bodies. Ann Neurol. 2017;81(6):898-903. https://doi.org/10.1002/a na.24937.

37. Mori F, Piao Y-S, Hayashi S, Fujiwara H, Hasegawa M, Yoshimoto M, et al. Alpha-synuclein accumulates in Purkinje cells in Lewy body disease but not in multiple system atrophy. J Neuropathol Exp Neurol. 2003;62(8):812-9. https://doi.org/10.1093/jnen/62.8.812.

38. Flønes $\mathbb{H}$, Fernandez-Vizarra E, Lykouri M, Brakedal B, Skeie GO, Miletic H, et al. Neuronal complex I deficiency occurs throughout the Parkinson's disease brain, but is not associated with neurodegeneration or mitochondrial DNA damage. Acta Neuropathol (Berl). 2018;135:409-25.

39. Karamanlidis G, Lee CF, Garcia-Menendez L, Kolwicz SC, Suthammarak W, Gong $\mathrm{G}$, et al. Mitochondrial complex I deficiency increases protein acetylation and accelerates heart failure. Cell Metab. 2013;18(2):239-50. https://doi.org/10.1016/j.cmet.2013.07.002

40. Singh P, Hanson PS, Morris CM. Sirtuin-2 Protects Neural Cells from Oxidative Stress and Is Elevated in Neurodegeneration. Park Dis. 2017;2017 [cited 2019 Aug 2] Available from: https://www.ncbi.nlm.nih.gov/pmc/a rticles/PMC5467326/.

41. Verdin E. NAD ${ }^{+}$in aging, metabolism, and neurodegeneration. Science. 2015;350(6265):1208-13. https://doi.org/10.1126/science.aac4854. 
42. Anderson KA, Madsen AS, Olsen CA, Hirschey MD. Metabolic control by sirtuins and other enzymes that sense NAD+, NADH, or their ratio. Biochim Biophys Acta. 1858;2017:991-8.

43. Sauve AA. Sirtuin chemical mechanisms. Biochim Biophys Acta. 2010;1804: 1591-603.

44. Imai S, Guarente L. It takes two to tango: NAD+ and sirtuins in aging/ longevity control. NPJ Aging Mech Dis. 2016;2(1):16017. https://doi.org/10.1 038/npjamd.2016.17.

45. Balan IS, Fiskum G, Kristian T. Visualization and quantification of $N A D(H)$ in brain sections by a novel histo-enzymatic nitrotetrazolium blue staining technique. Brain Res. 2010;1316C:112.

46. Braidy N, Guillemin GJ, Mansour H, Chan-Ling T, Poljak A, Grant R. Age related changes in NAD+ metabolism oxidative stress and Sirt1 activity in wistar rats. PLoS One. 2011;6(4):e19194. https://doi.org/10.1371/journal.pone. 0019194.

47. Koltai E, Szabo Z, Atalay M, Boldogh I, Naito H, Goto S, et al. Exercise alters SIRT1, SIRT6, NAD and NAMPT levels in skeletal muscle of aged rats. Mech Ageing Dev. 2010;131(1):21-8. https://doi.org/10.1016/j.mad.2009.11.002.

48. Allhoff M, Seré K, Pires JF, Zenke M, Costa IG. Differential peak calling of ChIP-seq signals with replicates with THOR. Nucleic Acids Res. 2016;44:e153.

49. Oldham MC, Konopka G, Iwamoto K, Langfelder P, Kato T, Horvath S, et al. Functional organization of the transcriptome in human brain. Nat Neurosci. 2008;11(11):1271-82. https://doi.org/10.1038/nn.2207.

50. Titus AJ, Gallimore RM, Salas LA, Christensen BC. Cell-type deconvolution from DNA methylation: a review of recent applications. Hum Mol Genet. 2017;26(R2):R216-24. https://doi.org/10.1093/hmg/ddx275.

51. Sun W, Poschmann J, Cruz-Herrera Del Rosario R, Parikshak NN, Hajan HS, Kumar V, et al. Histone Acetylome-wide Association Study of Autism Spectrum Disorder. Cell. 2016;167:1385-1397.e11.

52. Andrade-Moraes $\mathrm{CH}$, Oliveira-Pinto AV, Castro-Fonseca E, da Silva CG, Guimarães DM, Szczupak D, et al. Cell number changes in Alzheimer's disease relate to dementia, not to plaques and tangles. Brain J Neurol. 2013; 136(12):3738-52. https://doi.org/10.1093/brain/awt273.

53. von Bartheld CS, Bahney J, Herculano-Houzel S. The search for true numbers of neurons and glial cells in the human brain: a review of 150 years of cell counting. J Comp Neurol. 2016:524(18):3865-95. https://doi.org/10.1002/ cne.24040.

54. Gómez-Isla T, Price JL, McKeel DW, Morris JC, Growdon JH, Hyman BT. Profound loss of layer $\|$ entorhinal cortex neurons occurs in very mild Alzheimer's disease. J Neurosci. 1996;16(14):4491-500. https://doi.org/10.1 523/JNEUROSCI.16-14-04491.1996.

55. Price JL, Ko Al, Wade MJ, Tsou SK, McKeel DW, Morris JC. Neuron number in the Entorhinal cortex and CA1 in preclinical Alzheimer disease. Arch Neurol. 2001;58(9):1395-402. https://doi.org/10.1001/archneur.58.9.1395.

56. Wu H-C, Chen C-M, Chen Y-C, Fung H-C, Chang K-H, Wu Y-R. DLG2, but not TMEM229B, GPNMB, and ITGA8 polymorphism, is associated with Parkinson's disease in a Taiwanese population. Neurobiol Aging. 2018;64 158.e1-6.

57. Jansen IE, Ye H, Heetveld S, Lechler MC, Michels $H$, Seinstra Rl, et al. Discovery and functional prioritization of Parkinson's disease candidate genes from large-scale whole exome sequencing. Genome Biol. 2017;18(1): 22. https://doi.org/10.1186/s13059-017-1147-9.

58. Foo JN, Tan LC, Irwan ID, Au W-L, Low HQ, Prakash K-M, et al. Genome-wide association study of Parkinson's disease in east Asians. Hum Mol Genet. 2017;26(1):226-32. https://doi.org/10.1093/hmg/ddw379.

59. Schneider SA, Alcalay RN. Neuropathology of genetic synucleinopathies with parkinsonism: review of the literature. Mov Disord Off J Mov Disord Soc. 2017;32(11):1504-23. https://doi.org/10.1002/mds.27193.

60. Reed X, Bandrés-Ciga S, Blauwendraat C, Cookson MR. The role of monogenic genes in idiopathic Parkinson's disease. Neurobiol Dis. 2019;124: 230-9. https://doi.org/10.1016/j.nbd.2018.11.012.

61. Dickson DW. Neuropathology of Parkinson disease. Parkinsonism Relat Disord. 2018;46(Suppl 1):S30-3. https://doi.org/10.1016/j.parkreldis.2017.07. 033.

62. Lim EW, Aarsland D, Ffytche D, Taddei RN, van Wamelen DJ, Wan Y-M, et al. Amyloid- $\beta$ and Parkinson's disease. J Neurol. 2019;266(11):2605-19. https:// doi.org/10.1007/s00415-018-9100-8.

63. Soldner F, Stelzer Y, Shivalila CS, Abraham BJ, Latourelle JC, Barrasa MI, et al. Parkinson-associated risk variant in distal enhancer of a-synuclein modulates target gene expression. Nature. 2016;533(7601):95-9. https://doi.org/10.103 8/nature17939.
64. Mittal S, Bjornevik K, Im DS, Flierl A, Dong X, Locascio JJ, et al. beta2Adrenoreceptor is a regulator of the alpha-synuclein gene driving risk of Parkinson's disease. Science. 2017;357(6354):891-8. https://doi.org/10.1126/ science.aaf3934

65. Raisner R, Kharbanda S, Jin L, Jeng E, Chan E, Merchant M, et al. Enhancer activity requires CBP/P300 Bromodomain-dependent histone H3K27 acetylation. Cell Rep. 2018;24(7):1722-9. https://doi.org/10.1016/j.celrep.2018. 07.041.

66. Bouras T, Fu M, Sauve AA, Wang F, Quong AA, Perkins ND, et al. SIRT1 deacetylation and repression of p300 involves lysine residues 1020/1024 within the cell cycle regulatory domain 1. J Biol Chem. 2005;280(11):1026476. https://doi.org/10.1074/jbc.M408748200.

67. Karlić R, Chung H-R, Lasserre J, Vlahoviček K, Vingron M. Histone modification levels are predictive for gene expression. Proc Natl Acad Sci U S A. 2010;107(7):2926-31. https://doi.org/10.1073/pnas.0909344107.

68. Scott GK, Mattie MD, Berger CE, Benz SC, Benz CC. Rapid alteration of microRNA levels by histone deacetylase inhibition. Cancer Res. 2006;66(3): 1277-81. https://doi.org/10.1158/0008-5472.CAN-05-3632.

69. Lopez-Atalaya JP, Ito S, Valor LM, Benito E, Barco A. Genomic targets, and histone acetylation and gene expression profiling of neural HDAC inhibition. Nucleic Acids Res. 2013;41(17):8072-84. https://doi.org/10.1093/na r/gkt590.

70. Creyghton MP, Cheng AW, Welstead GG, Kooistra T, Carey BW, Steine EJ, et al. Histone H3K27ac separates active from poised enhancers and predicts developmental state. Proc Natl Acad Sci. 2010;107(50):21931-6. https://doi. org/10.1073/pnas.1016071107.

71. Ernst J, Kheradpour P, Mikkelsen TS, Shoresh N, Ward LD, Epstein CB, et al. Mapping and analysis of chromatin state dynamics in nine human cell types. Nature. 2011;473(7345):43-9. https://doi.org/10.1038/nature09906.

72. Cantó C, Menzies K, Auwerx J. NAD+ metabolism and the control of energy homeostasis - a balancing act between mitochondria and the nucleus. Cell Metab. 2015;22(1):31-53. https://doi.org/10.1016/j.cmet.2015.05.023.

73. Schapira AH, Cooper JM, Dexter D, Jenner P, Clark JB, Marsden CD. Mitochondrial complex I deficiency in Parkinson's disease. Lancet. 1989;1: 1269

74. Huang M, Lou D, Charli A, Kong D, Jin H, Anantharam V, et al. Mitochondrial Dysfunction Induces Epigenetic Dysregulation by H3K27 Hyperacetylation to Perturb Active Enhancers in Parkinson's Disease Models. bioRxiv. 2019: 808246. https://doi.org/10.1101/808246.

75. Schwab AJ, Sison SL, Meade MR, Broniowska KA, Corbett JA, Ebert AD. Decreased Sirtuin Deacetylase activity in LRRK2 G2019S iPSC-derived dopaminergic neurons. Stem Cell Rep. 2017;9(6):1839-52. https://doi.org/1 0.1016/j.stemcr.2017.10.010.

76. Pickrell AM, Youle RJ. The roles of PINK1, parkin, and mitochondrial fidelity in Parkinson's disease. Neuron. 2015;85(2):257-73. https://doi.org/10.1016/j. neuron.2014.12.007.

77. Polymeropoulos MH, Lavedan C, Leroy E, Ide SE, Dehejia A, Dutra A, et al. Mutation in the alpha-synuclein gene identified in families with Parkinson's disease. Science. 1997;276(5321):2045-7. https://doi.org/10.1126/science.276. 5321.2045 .

78. Shojaee S, Sina F, Banihosseini SS, Kazemi MH, Kalhor R, Shahidi G-A, et al. Genome-wide linkage analysis of a Parkinsonian-pyramidal syndrome pedigree by 500 K SNP arrays. Am J Hum Genet. 2008;82(6):1375-84. https:// doi.org/10.1016/j.ajhg.2008.05.005.

79. Yang YX, Muqit MMK, Latchman DS. Induction of parkin expression in the presence of oxidative stress. Eur J Neurosci. 2006;24(5):1366-72. https://doi. org/10.1111/j.1460-9568.2006.04998.x.

80. Bouman L, Schlierf A, Lutz AK, Shan J, Deinlein A, Kast J, et al. Parkin is transcriptionally regulated by ATF4: evidence for an interconnection between mitochondrial stress and ER stress. Cell Death Differ. 2011;18(5): 769-82. https://doi.org/10.1038/cdd.2010.142.

81. Baulac S, Lu H, Strahle J, Yang T, Goldberg MS, Shen J, et al. Increased DJ-1 expression under oxidative stress and in Alzheimer's disease brains. Mol Neurodegener. 2009;4(1):12. https://doi.org/10.1186/1750-1326-4-12.

82. Puspita L, Chung SY, Shim J-W. Oxidative stress and cellular pathologies in Parkinson's disease. Mol Brain. 2017;10(1):53. https://doi.org/10.1186/s13041017-0340-9.

83. Nair N, Shoaib M, Sørensen CS. Chromatin Dynamics in Genome Stability: Roles in Suppressing Endogenous DNA Damage and Facilitating DNA Repair. Int J Mol Sci. 2017;18 [cited 2020 Mar 23] Available from: https:// www.ncbi.nlm.nih.gov/pmc/articles/PMC5535976/. 
84. van Heesbeen HJ, Smidt MP. Entanglement of Genetics and Epigenetics in Parkinson's Disease. Front Neurosci. 2019;13 [cited 2020 Feb 5]; Available from: https://www.frontiersin.org/articles/10.3389/fnins.2019.00277/full.

85. Lecellier $\mathrm{CH}$, Wasserman WW, Mathelier A. Human Enhancers Harboring Specific Sequence Composition, Activity, and Genome Organization Are Linked to the Immune Response. Genetics. 2018;209:1055-71.

86. Anderson RM, Barger JL, Edwards MG, Braun KH, O'Connor CE, Prolla TA, et al. Dynamic regulation of PGC-1a localization and turnover implicates mitochondrial adaptation in calorie restriction and the stress response. Aging Cell. 2008;7(1):101-11. https://doi.org/10.1111/j.1474-9726.2007.00357.x.

\section{Publisher's Note}

Springer Nature remains neutral with regard to jurisdictional claims in published maps and institutional affiliations.

Ready to submit your research? Choose BMC and benefit from:

- fast, convenient online submission

- thorough peer review by experienced researchers in your field

- rapid publication on acceptance

- support for research data, including large and complex data types

- gold Open Access which fosters wider collaboration and increased citations

- maximum visibility for your research: over $100 \mathrm{M}$ website views per year

At BMC, research is always in progress.

Learn more biomedcentral.com/submissions 